\title{
EXPANSION OF ITERATED STRATONOVICH STOCHASTIC INTEGRALS BASED ON GENERALIZED MULTIPLE FOURIER SERIES
}

\author{
D.F. KUZNETSOV
}

\begin{abstract}
The article is devoted to expansions of iterated Stratonovich stochastic integrals of multiplicities 1-4 on the base of the method of generalized multiple Fourier series. We prove the mean-square convergence of expansions in the case of Legendre polynomials as well as in the case of trigonometric functions. The considered expansions contain only one passage to the limit in contrast to its existing analogues. This property is very convenient for the mean-square approximation of iterated stochastic integrals. It is well-known that a prospective approach to numerical solving of Itô stochastic differential equations being adequate mathematical models of dynamical systems of various physical nature is one based on stochastic analogue of Taylor formula for the solutions to these equations. The iterated stochastic Stratonovich integrals are parts of so-called Taylor-Stratonovich expansion being one of the aforementioned stochastic analogues of Taylor formula. This is why the results of the paper can be applied to constructing strong numerical methods of convergence orders 1.0, 1.5 and 2.0 for Itô stochastic differential equations. The method of generalized multiple Fourier series does not require a partitioning of the integration interval for iterated stochastic Stratonovich integrals. This feature is essential since the mentioned integration interval is small playing a role of the integration step in numerical methods for Itô stochastic differential equations.
\end{abstract}

Keywords: iterated Stratonovich stochastic integral, multiple Fourier series, Legendre polynomial, expansion, mean-square convergence.

Mathematics Subject Classification: 60H05

\section{INTRODUCTION}

Suppose that we are given a fixed probability space $(\Omega, \mathrm{F}, \mathrm{P})$, a non-decreasing set of $\sigma$ algebras $\left\{\mathrm{F}_{t}, t \in[0, T]\right\}$ on this space and a $\mathrm{F}_{t}$-measurable for all $t \in[0, T] m$-dimensional Wiener process $\mathbf{f}_{t}$ with independent components $\mathbf{f}_{t}^{(i)}, i=1, \ldots, m$, and the process $\mathbf{f}_{t+\Delta}-\mathbf{f}_{t}$ is independent of the events in the $\sigma$-algebra $\mathrm{F}_{t}$ for all $t \geqslant 0, \Delta>0$. We assume that the $\sigma$-algebra $\mathrm{F}$ is complete with respect to the measure $\mathrm{P}$, while the $\sigma$-algebra $\mathrm{F}_{0}$ contains all events of zero probability.

We consider a Itô stochastic differential equation (SDE)

$$
\mathbf{x}_{t}=\mathbf{x}_{0}+\int_{0}^{t} \mathbf{a}\left(\mathbf{x}_{\tau}, \tau\right) d \tau+\int_{0}^{t} B\left(\mathbf{x}_{\tau}, \tau\right) d \mathbf{f}_{\tau}, \quad \mathbf{x}_{0}=\mathbf{x}(0, \omega), \quad \omega \in \Omega .
$$

Here $\mathbf{x}_{\tau}, \tau \in[0, T]$, is a $n$-dimensional random process being a strong solution of Itô SDE (1), the second integral in the right hand side (1) is treated as a Itô stochastic integral, a :

D.F. Kuznetsov, Expansion of iterated Stratonovich Stochastic Integrals Based ON GENERALIZED MULTIPLE FOURIER SERIES.

C.Kuznetsov D.F. 2019.

Submitted September 1, 2018. 
$\mathbb{R}^{n} \times[0, T] \rightarrow \mathbb{R}^{n}, B: \mathbb{R}^{n} \times[0, T] \rightarrow \mathbb{R}^{n \times m}$ are deterministic functions, for the right hand side in (1) is well-defined and satisfies standard conditions of existence and uniqueness of a strong solution $\mathbf{x}_{\tau}, \tau \in[0, T]$, to Itô SDE (1) [1], $\mathbf{x}_{0}$ and $\mathbf{f}_{\tau}-\mathbf{f}_{0}, \tau>0$, are assumed to be independent and $\mathbf{x}_{0}$ is a $n$-dimensional $\mathrm{F}_{0}$-measurable random variable obeying $\operatorname{M}\left\{\left|\mathbf{x}_{0}\right|^{2}\right\}<\infty, M$ is the expectation operator.

It is known [2]-[4] that one of the prospective approaches to a numerical integration of Itô SDE is one based on stochastic analogues of the Taylor formula for solutions to these equations. This approach employs a finite discretization of a time variable and supposes a numerical modelling of the solution to Itô SDE at discrete times by means of stochastic analogue of Taylor formula.

An important feature of the stochastic analogues of Taylor formula [2]-[11] for solutions to Itô SDE is that they involve so-called Itô or Stratonovich iterated stochastic integrals (ISI), which are functionals of the components of the Wiener process and have a complicated structure.

In one of the most general form, the mentioned Itô and Stratonovich ISIs read as

$$
\begin{aligned}
J\left[\psi^{(k)}\right]_{T, t} & =\int_{t}^{T} \psi_{k}\left(t_{k}\right) \ldots \int_{t}^{t_{2}} \psi_{1}\left(t_{1}\right) d \mathbf{w}_{t_{1}}^{\left(i_{1}\right)} \ldots d \mathbf{w}_{t_{k}}^{\left(i_{k}\right)}, \\
J^{*}\left[\psi^{(k)}\right]_{T, t} & =\int_{t}^{* T} \psi_{k}\left(t_{k}\right) \ldots \int_{t}^{* t_{2}} \psi_{1}\left(t_{1}\right) d \mathbf{w}_{t_{1}}^{\left(i_{1}\right)} \ldots d \mathbf{w}_{t_{k}}^{\left(i_{k}\right)},
\end{aligned}
$$

where $\psi_{l}(\tau), l=1, \ldots, k$, are continuous on the segment $[t, T]$ deterministic functions, $\mathbf{w}_{\tau}$ is a random vector with $m+1$ components of the form: $\mathbf{w}_{\tau}^{(i)}=\mathbf{f}_{\tau}^{(i)}$ as $i=1, \ldots, m$ and $\mathbf{w}_{\tau}^{(0)}=\tau$, the quantities $i_{1}, \ldots, i_{k}$ takes values $0,1, \ldots, m, \mathbf{f}_{\tau}^{(i)}, i=1, \ldots, m$ are independent standard Wiener processes, $k$ is the multiplicity of ISI. In (2) and (3), and also in what follows, to simplify the writing, instead of $J\left[\psi^{(k)}\right]_{T, t}^{i_{1} \ldots i_{k}}$ and $J^{*}\left[\psi^{(k)}\right]_{T, t}^{u_{1} \ldots i_{k}}$ we write $J\left[\psi^{(k)}\right]_{T, t}$ and $J^{*}\left[\psi^{(k)}\right]_{T, t}$, respectively.

We note that classical stochastic analogues of Taylor formula, so-called Taylor-Itô and TaylorStratonovich expansions [2]-[6] involve respectively Itô and Stratonovich ISIs of form (2) and (3) as $\psi_{1}(\tau), \ldots, \psi_{k}(\tau) \equiv 1$ and $i_{1}, \ldots, i_{k}=0,1, \ldots, m$.

Transformed analogues of the above expansions, so-called unified Taylor-Itô and TaylorStratonovich expansions [7], [8] involve respectively Itô and Stratonovich ISIs of form (2) and (3) as $\psi_{l}(\tau) \equiv(t-\tau)^{q_{l}}, \tau \in[t, T]$ and $i_{l}=1, \ldots, m, q_{l}=0,1,2 \ldots, l=1, \ldots, k$.

In view of the said above, the systems of Itô and Stratonovich ISIs play an exceptionally important role in resolving the problem on numerical integration of Itô SDEs.

At first glance, it could seem that ISIs can be approximated by iterated integral sums. However, such approach supposes a partitioning of the integration interval $[t, T]$ for ISI and its length $T-t$ is already rather small since it serves as an integration step in numerical methods for Itô SDE. As numerical experiments show [9], this leads one to unacceptable computational costs.

In [3], it was proposed to employ mean-square converging trigonometric Fourier expansions for Wiener processes, by which ISI is constructed. In [3], this method was employed to obtain expansions for Itô ISI of form (2) as $k=2$ and $\psi_{1}(\tau), \psi_{2}(\tau) \equiv 1, i_{1}, i_{2}=0,1, \ldots, m$.

An attempt to develop this idea for Stratonovich ISI of form (3) as $k=3$ and $\psi_{1}(\tau), \psi_{2}(\tau), \psi_{3}(\tau) \equiv 1, i_{1}, i_{2}, i_{3}=0,1, \ldots, m$ was made in [2], [10]

In [11] there was proposed a more general method of mean-square approximation of Stratonovich ISI of form (3) based on generalized iteratied Fourier series, which allows one to employ complete orthonormal systems of Legendre polynomials and trigonometric functions in the space $L_{2}([t, T])$, due to its features, the method used in [3] admits the application of only trigonometric basis functions. 
The methods employing the Fourier series and being proposed in [2]-[4], [10], [11] turned out to be essentially more effective for a mean-square approximation of Stratonovich and Itô ISIs than the methods based on integral sums [9]. However, the Fourier methods considered in [2]-[4], [10], [11] lead one to iterated series of standard Gaussian random variables, in which the passage to the limit is made iteratively. This is opposite to the multiple series, in which the passage to the limit is made just once. This fact is essential and gives rise to a series of restrictions for the application of the methods from [2]-[4], [10], [11] to ISIs of form (2) and (3) of multiplicity 3 and higher, here we mean at least three-multiple integration over Wiener processes in ISI.

In [9], there was proposed a method of mean-square approximation for Itô ISI of form (2) (see Theorem 1 in what follows) based on multiple (not iterated) generalized Fourier series over various complete orthonormal systems of basis functions in the space $L_{2}\left([t, T]^{k}\right)$. As a result, in the mentioned method, the passage to the limit is made just once that ensures a correct choice of the lengths for the sequences of standard Gaussian random variables needed for constructing an approximation of Itô ISI. Moreover, the Fourier method provides new opportunities for estimating and exact calculation of mean-square errors in approximations for Itô ISI [9].

The present paper is devoted to an adaption of the Fourier method [9] of expansion of Itô ISI of form (2) to Stratonovich ISI of form (3). In the work we show that the expansions for Stratonovich ISIs of form (3) obtained by the method in [9] turn out to be essentially simpler and having no additional rather complicated terms in comparison with its analogues obtained first in [9] for Itô ISI of form (2).

\section{Formulation of main Results}

We provide a formulation of Fourier method [9].

Let $\left\{\phi_{j}(x)\right\}_{j=0}^{\infty}$ be a complete orthonormal system of functions in the space $L_{2}([t, T])$, and $\psi_{1}(\tau), \ldots, \psi_{k}(\tau)$ be deterministic functions continuous on $[t, T]$. We introduce the following function:

$$
K\left(t_{1}, \ldots, t_{k}\right)=\psi_{1}\left(t_{1}\right) \ldots \psi_{k}\left(t_{k}\right) \mathbf{1}_{\left\{t_{1}<\ldots<t_{k}\right\}}, \quad t_{1}, \ldots, t_{k} \in[t, T], \quad k \geqslant 2,
$$

and $K\left(t_{1}\right)=\psi_{1}\left(t_{1}\right), t_{1} \in[t, T]$, where $\mathbf{1}_{\{A\}}$ is the indicator of the set $A$.

The function $K\left(t_{1}, \ldots, t_{k}\right)$ is piece-wise continuous in the hyper-cube $[t, T]^{k}$ and this is why a multiple Fourier series of the function $K\left(t_{1}, \ldots, t_{k}\right) \in L_{2}\left([t, T]^{k}\right)$ converges in the hyper-cube $[t, T]^{k}$ in the square-mean sense, that is,

$$
\lim _{p_{1}, \ldots, p_{k} \rightarrow \infty} \int_{[t, T]^{k}}\left(K\left(t_{1}, \ldots, t_{k}\right)-\sum_{j_{1}=0}^{p_{1}} \ldots \sum_{j_{k}=0}^{p_{k}} C_{j_{k} \ldots j_{1}} \prod_{l=1}^{k} \phi_{j_{l}}\left(t_{l}\right)\right)^{2} d t_{1} \ldots d t_{k}=0
$$

where

$$
C_{j_{k} \ldots j_{1}}=\int_{[t, T]^{k}} K\left(t_{1}, \ldots, t_{k}\right) \prod_{l=1}^{k} \phi_{j_{l}}\left(t_{l}\right) d t_{1} \ldots d t_{k}
$$

and the Parseval identity holds:

$$
\int_{[t, T]^{k}} K^{2}\left(t_{1}, \ldots, t_{k}\right) d t_{1} \ldots d t_{k}=\lim _{p_{1}, \ldots, p_{k} \rightarrow \infty} \sum_{j_{1}=0}^{p_{1}} \ldots \sum_{j_{k}=0}^{p_{k}}\left(C_{j_{k} \ldots j_{1}}\right)^{2} .
$$

We consider a partition $\left\{\tau_{j}\right\}_{j=0}^{N}$ of the segment $[t, T]$ such that

$$
t=\tau_{0}<\ldots<\tau_{N}=T, \quad \Delta_{N}=\max _{0 \leqslant j \leqslant N-1} \Delta \tau_{j} \rightarrow 0 \quad \text { as } \quad N \rightarrow \infty, \quad \Delta \tau_{j}=\tau_{j+1}-\tau_{j} .
$$


Theorem 1. [9] Let $\left\{\phi_{j}(x)\right\}_{j=0}^{\infty}$ be a complete orthonormal system of continuous functions in the space $L_{2}([t, T])$, and $\psi_{i}(\tau), i=1,2, \ldots, k$, are continuous on the segment $[t, T]$ functions. Then Itô ISI $J\left[\psi^{(k)}\right]_{T, t}$ of form (2) is expanded into a multiple series converging in the meansquare sense:

$$
\begin{aligned}
J\left[\psi^{(k)}\right]_{T, t}= & \underset{p_{1}, \ldots, p_{k} \rightarrow \infty}{\lim } \sum_{j_{1}=0}^{p_{1}} \ldots \sum_{j_{k}=0}^{p_{k}} C_{j_{k} \ldots j_{1}}\left(\prod_{l=1}^{k} \zeta_{j_{l}}^{\left(i_{l}\right)}\right. \\
& \left.-\underset{N \rightarrow \infty}{\operatorname{li} . \mathrm{m} .} \sum_{\left(l_{1}, \ldots, l_{k}\right) \in G_{k}} \phi_{j_{1}}\left(\tau_{l_{1}}\right) \Delta \mathbf{w}_{\tau_{l_{1}}}^{\left(i_{1}\right)} \ldots \phi_{j_{k}}\left(\tau_{l_{k}}\right) \Delta \mathbf{w}_{\tau_{l_{k}}}^{\left(i_{k}\right)}\right),
\end{aligned}
$$

where l.i.m. is the limit in the mean-square sense,

$$
\begin{aligned}
& G_{k}=H_{k} \backslash L_{k}, \quad H_{k}=\left\{\left(l_{1}, \ldots, l_{k}\right): l_{1}, \ldots, l_{k}=0,1, \ldots, N-1\right\}, \\
& L_{k}=\left\{\left(l_{1}, \ldots, l_{k}\right): l_{1}, \ldots, l_{k}=0,1, \ldots, N-1, l_{g} \neq l_{r}(g \neq r), g, r=1, \ldots, k\right\},
\end{aligned}
$$

and

$$
\zeta_{j}^{(i)}=\int_{t}^{T} \phi_{j}(s) d \mathbf{w}_{s}^{(i)}
$$

are independent standard Gaussian random variables for different $i$ or $j$ if $i \neq 0$, and $\Delta \mathbf{w}_{\tau_{j}}^{(i)}=$ $\mathbf{w}_{\tau_{j+1}}^{(i)}-\mathbf{w}_{\tau_{j}}^{(i)}, i=0,1, \ldots, m,\left\{\tau_{j}\right\}_{j=0}^{N}$ is a partition of the segment $[t, T]$ satisfying condition (7).

It is easy to show that particular cases (8) as $k=1, \ldots, 4$ are written as

$$
\begin{aligned}
& J\left[\psi^{(1)}\right]_{T, t}=\underset{p_{1} \rightarrow \infty}{\lim . \sum_{j_{1}=0}^{p_{1}}} C_{j_{1}} \zeta_{j_{1}}^{\left(i_{1}\right)} \\
& J\left[\psi^{(2)}\right]_{T, t}=\underset{p_{1}, p_{2} \rightarrow \infty}{\operatorname{l.i.m}} \sum_{j_{1}=0}^{p_{1}} \sum_{j_{2}=0}^{p_{2}} C_{j_{2} j_{1}}\left(\zeta_{j_{1}}^{\left(i_{1}\right)} \zeta_{j_{2}}^{\left(i_{2}\right)}-\mathbf{1}_{\left\{i_{1}=i_{2} \neq 0\right\}} \mathbf{1}_{\left\{j_{1}=j_{2}\right\}}\right),
\end{aligned}
$$

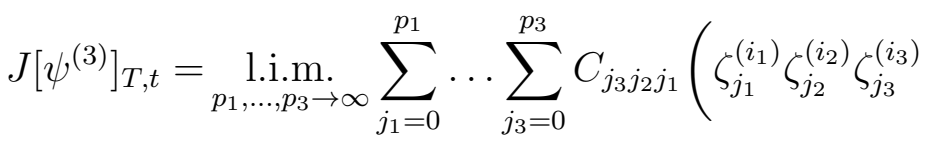

$$
\begin{aligned}
& \left.-\mathbf{1}_{\left\{i_{1}=i_{2} \neq 0\right\}} \mathbf{1}_{\left\{j_{1}=j_{2}\right\}} \zeta_{j_{3}}^{\left(i_{3}\right)}-\mathbf{1}_{\left\{i_{2}=i_{3} \neq 0\right\}} \mathbf{1}_{\left\{j_{2}=j_{3}\right\}} \zeta_{j_{1}}^{\left(i_{1}\right)}-\mathbf{1}_{\left\{i_{1}=i_{3} \neq 0\right\}} \mathbf{1}_{\left\{j_{1}=j_{3}\right\}} \zeta_{j_{2}}^{\left(i_{2}\right)}\right), \\
& J\left[\psi^{(4)}\right]_{T, t}=\underset{p_{1}, \ldots, p_{4} \rightarrow \infty}{\operatorname{l.i.m.}} \sum_{j_{1}=0}^{p_{1}} \ldots \sum_{j_{4}=0}^{p_{4}} C_{j_{4} \ldots j_{1}}\left(\prod_{l=1}^{4} \zeta_{j_{l}}^{\left(i_{l}\right)}-\mathbf{1}_{\left\{i_{1}=i_{2} \neq 0\right\}} \mathbf{1}_{\left\{j_{1}=j_{2}\right\}} \zeta_{j_{3}}^{\left(i_{3}\right)} \zeta_{j_{4}}^{\left(i_{4}\right)}\right. \\
& -\mathbf{1}_{\left\{i_{1}=i_{3} \neq 0\right\}} \mathbf{1}_{\left\{j_{1}=j_{3}\right\}} \zeta_{j_{2}}^{\left(i_{2}\right)} \zeta_{j_{4}}^{\left(i_{4}\right)}-\mathbf{1}_{\left\{i_{1}=i_{4} \neq 0\right\}} \mathbf{1}_{\left\{j_{1}=j_{4}\right\}} \zeta_{j_{2}}^{\left(i_{2}\right)} \zeta_{j_{3}}^{\left(i_{3}\right)} \\
& -\mathbf{1}_{\left\{i_{2}=i_{3} \neq 0\right\}} \mathbf{1}_{\left\{j_{2}=j_{3}\right\}} \zeta_{j_{1}}^{\left(i_{1}\right)} \zeta_{j_{4}}^{\left(i_{4}\right)}-\mathbf{1}_{\left\{i_{2}=i_{4} \neq 0\right\}} \mathbf{1}_{\left\{j_{2}=j_{4}\right\}} \zeta_{j_{1}}^{\left(i_{1}\right)} \zeta_{j_{3}}^{\left(i_{3}\right)} \\
& -\mathbf{1}_{\left\{i_{3}=i_{4} \neq 0\right\}} \mathbf{1}_{\left\{j_{3}=j_{4}\right\}} \zeta_{j_{1}}^{\left(i_{1}\right)} \zeta_{j_{2}}^{\left(i_{2}\right)}+\mathbf{1}_{\left\{i_{1}=i_{2} \neq 0\right\}} \mathbf{1}_{\left\{j_{1}=j_{2}\right\}} \mathbf{1}_{\left\{i_{3}=i_{4} \neq 0\right\}} \mathbf{1}_{\left\{j_{3}=j_{4}\right\}} \\
& \left.+\mathbf{1}_{\left\{i_{1}=i_{3} \neq 0\right\}} \mathbf{1}_{\left\{j_{1}=j_{3}\right\}} \mathbf{1}_{\left\{i_{2}=i_{4} \neq 0\right\}} \mathbf{1}_{\left\{j_{2}=j_{4}\right\}}+\mathbf{1}_{\left\{i_{1}=i_{4} \neq 0\right\}} \mathbf{1}_{\left\{j_{1}=j_{4}\right\}} \mathbf{1}_{\left\{i_{2}=i_{3} \neq 0\right\}} \mathbf{1}_{\left\{j_{2}=j_{3}\right\}}\right) \text {. }
\end{aligned}
$$

We are in position to formulate the main results of the work, which show that analogues of expansions (10)-(12) obtained for Stratonovich ISI of form (3) turn out to be essentially simpler than expansions $10-12$.

Theorem 2. Let $\left\{\phi_{j}(x)\right\}_{j=0}^{\infty}$ be a complete orthonormalized system of Legendre polynomials or a system of trigonometric functions in the space $L_{2}([t, T])$. Assume also that a function $\psi_{2}(\tau)$ 
is continuously differentiable on the segment $[t, T]$, and a function $\psi_{1}(\tau)$ is twice continuously differentiable on this segment. Then the Stratonovich ISI $J^{*}\left[\psi^{(2)}\right]_{T, t}$ of multiplicity 2 of form (3) with $i_{1}, i_{2}=1, \ldots, m$ satisfies the following converging in the square-mean sense expansion

$$
J^{*}\left[\psi^{(2)}\right]_{T, t}=\underset{p_{1}, p_{2} \rightarrow \infty}{\lim . \sum_{j_{1}=0}^{p_{1}}} \sum_{j_{2}=0}^{p_{2}} C_{j_{2} j_{1}} \zeta_{j_{1}}^{\left(i_{1}\right)} \zeta_{j_{2}}^{\left(i_{2}\right)},
$$

where the notations are the same as in Theorem 1.

Theorem 3. Let $\left\{\phi_{j}(x)\right\}_{j=0}^{\infty}$ be a complete orthonormal system of Legendre polynomials or a system of trigonometric functions in the space $L_{2}([t, T])$. Assume also that a function $\psi_{2}(s)$ is continuously differentiable on the segment $[t, T]$, and function $\psi_{1}(s), \psi_{3}(s)$ are twice continuously differentiable on this segment. Then the Stratonovich ISI $J^{*}\left[\psi^{(3)}\right]_{T, t}$ of multiplicity 3 of form (3) with $i_{1}, i_{2}, i_{3}=1, \ldots, m$ satisfies the following converging in the square-mean sense expansion

$$
J^{*}\left[\psi^{(3)}\right]_{T, t}=\underset{p \rightarrow \infty}{\lim .} \sum_{j_{1}, j_{2}, j_{3}=0}^{p} C_{j_{3} j_{2} j_{1}} \zeta_{j_{1}}^{\left(i_{1}\right)} \zeta_{j_{2}}^{\left(i_{2}\right)} \zeta_{j_{3}}^{\left(i_{3}\right)},
$$

where the notations are the same as in Theorem 1.

Theorem 4. Let $\left\{\phi_{j}(x)\right\}_{j=0}^{\infty}$ be a complete orthonormal system of Legendre polynomials or a system of trigonometric functions in the space $L_{2}([t, T])$. Assume also that $\psi_{1}(s), \ldots, \psi_{4}(s) \equiv 1$. Then the Stratonovich ISI $J^{*}\left[\psi^{(4)}\right]_{T, t}$ of multiplicity 4 of form (3) with $i_{1}, i_{2}, i_{3}, i_{4}=0,1, \ldots, m$ satisfies the following converging in the square-mean sense expansion

$$
J^{*}\left[\psi^{(4)}\right]_{T, t}=\underset{p \rightarrow \infty}{\lim . \mathrm{m}} \sum_{j_{1}, j_{2}, j_{3}, j_{4}=0}^{p} C_{j_{4} j_{3} j_{2} j_{1}} \zeta_{j_{1}}^{\left(i_{1}\right)} \zeta_{j_{2}}^{\left(i_{2}\right)} \zeta_{j_{3}}^{\left(i_{3}\right)} \zeta_{j_{4}}^{\left(i_{4}\right)},
$$

where the notations are the same as in Theorem 1.

\section{Proof of TheOREM 2}

In view of the standard relation of Stratonovich and Itô integrals [2], the identity holds

$$
J^{*}\left[\psi^{(2)}\right]_{T, t}=J\left[\psi^{(2)}\right]_{T, t}+\frac{1}{2} \mathbf{1}_{\left\{i_{1}=i_{2} \neq 0\right\}} \int_{t}^{T} \psi_{1}\left(t_{1}\right) \psi_{2}\left(t_{1}\right) d t_{1}
$$

with probability 1.

According (10), (15), Theorem 2 will be proved if we show that

$$
\frac{1}{2} \int_{t}^{T} \psi_{1}\left(t_{1}\right) \psi_{2}\left(t_{1}\right) d t_{1}=\sum_{j_{1}=0}^{\infty} C_{j_{1} j_{1}} .
$$

We consider the function

$$
K^{*}\left(t_{1}, t_{2}\right)=K\left(t_{1}, t_{2}\right)+\frac{1}{2} \mathbf{1}_{\left\{t_{1}=t_{2}\right\}} \psi_{1}\left(t_{1}\right) \psi_{2}\left(t_{1}\right),
$$

where $t_{1}, t_{2} \in[t, T]$, and $K\left(t_{1}, t_{2}\right)$ is of form (4) as $k=2$.

We expand the function $K^{*}\left(t_{1}, t_{2}\right)$ into the Fourier series on the interval $(t, T)$ with respect to the variable $t_{1}$ for a fixed $t_{2}$ :

$$
K^{*}\left(t_{1}, t_{2}\right)=\sum_{j_{1}=0}^{\infty} C_{j_{1}}\left(t_{2}\right) \phi_{j_{1}}\left(t_{1}\right), \quad t_{1} \neq t, \quad t_{1} \neq T,
$$


where

$$
C_{j_{1}}\left(t_{2}\right)=\int_{t}^{T} K^{*}\left(t_{1}, t_{2}\right) \phi_{j_{1}}\left(t_{1}\right) d t_{1}=\psi_{2}\left(t_{2}\right) \int_{t}^{t_{2}} \psi_{1}\left(t_{1}\right) \phi_{j_{1}}\left(t_{1}\right) d t_{1} .
$$

Identity 18$)$ is satisfied at each point in the interval $(t, T)$ with respect to the variable $t_{1}$ for a fixed $t_{2}$ thanks to a piece-wise smoothness of the function $K^{*}\left(t_{1}, t_{2}\right)$ in the variable $t_{1}$ [12]-[14]. We also note that according the well-known properties of the Fourier series [12]-[14], series (18) converges as $t_{1}=t$ and $t_{1}=T$. While obtaining (18), we have also employed the fact [12]--14] that the right hand side of (18) converges as $t_{1}=t_{2}$, which is a point of a finite jump of the function $K^{*}\left(t_{1}, t_{2}\right)$, to the quantity

$$
\frac{1}{2}\left(K^{*}\left(t_{2}-0, t_{2}\right)+K^{*}\left(t_{2}+0, t_{2}\right)\right)=\frac{1}{2} \psi_{1}\left(t_{2}\right) \psi_{2}\left(t_{2}\right)=K^{*}\left(t_{2}, t_{2}\right) .
$$

The function $C_{j_{1}}\left(t_{2}\right)$ is continuously differentiable. We expand it into the Fourier series on the interval $(t, T)$ :

$$
C_{j_{1}}\left(t_{2}\right)=\sum_{j_{2}=0}^{\infty} C_{j_{2} j_{1}} \phi_{j_{2}}\left(t_{2}\right), \quad t_{2} \neq t, \quad t_{2} \neq T
$$

where $C_{j_{2} j_{1}}$ is of form $(6)$ as $k=2$, while identity $(19)$ is satisfied at each point in the interval $(t, T)$, the right hand side of $(19)$ converges as $t_{2}=t, t_{2}=T[12]-[14]$.

We substitute (19) into (18):

$$
K^{*}\left(t_{1}, t_{2}\right)=\sum_{j_{1}=0}^{\infty} \sum_{j_{2}=0}^{\infty} C_{j_{2} j_{1}} \phi_{j_{1}}\left(t_{1}\right) \phi_{j_{2}}\left(t_{2}\right), \quad\left(t_{1}, t_{2}\right) \in(t, T)^{2} .
$$

It is easy to see that letting $t_{1}=t_{2}$ in $(20)$, we obtain:

$$
\frac{1}{2} \psi_{1}\left(t_{1}\right) \psi_{2}\left(t_{1}\right)=\sum_{j_{1}=0}^{\infty} \sum_{j_{2}=0}^{\infty} C_{j_{2} j_{1}} \phi_{j_{1}}\left(t_{1}\right) \phi_{j_{2}}\left(t_{1}\right) .
$$

By means 21) we can formally write:

$$
\begin{aligned}
\frac{1}{2} \int_{t}^{T} \psi_{1}\left(t_{1}\right) \psi_{2}\left(t_{1}\right) d t_{1} & =\int_{t}^{T} \sum_{j_{1}=0}^{\infty} \sum_{j_{2}=0}^{\infty} C_{j_{2} j_{1}} \phi_{j_{1}}\left(t_{1}\right) \phi_{j_{2}}\left(t_{1}\right) d t_{1} \\
& =\sum_{j_{1}=0}^{\infty} \sum_{j_{2}=0}^{\infty} \int_{t}^{T} C_{j_{2} j_{1}} \phi_{j_{1}}\left(t_{1}\right) \phi_{j_{2}}\left(t_{1}\right) d t_{1} \\
& =\lim _{p_{1} \rightarrow \infty} \lim _{p_{2} \rightarrow \infty} \sum_{j_{1}=0}^{p_{1}} \sum_{j_{2}=0}^{p_{2}} C_{j_{2} j_{1}} \int_{t}^{T} \phi_{j_{1}}\left(t_{1}\right) \phi_{j_{2}}\left(t_{1}\right) d t_{1} \\
& =\lim _{p_{1} \rightarrow \infty} \lim _{p_{2} \rightarrow \infty} \sum_{j_{1}=0}^{p_{1}} \sum_{j_{2}=0}^{p_{2}} C_{j_{2} j_{1}} \mathbf{1}_{\left\{j_{1}=j_{2}\right\}} \\
& =\lim _{p_{1} \rightarrow \infty} \lim _{p_{2} \rightarrow \infty} \sum_{j_{1}=0}^{\min \left\{p_{1}, p_{2}\right\}} C_{j_{2} j_{1}}=\sum_{j_{1}=0}^{\infty} C_{j_{1} j_{1}} .
\end{aligned}
$$

In what follows, by $C, K, C_{0}, K_{0}, C_{1}, K_{1}, \ldots$ we denote various constants. 
Let us clarify how we pass from the first line in $(22)$ to the second one, other arguing in (22) follows the orthonormality of the functions $\phi_{j}(s)$ on the segment $[t, T]$. We have:

$$
\begin{aligned}
\left|\int_{t}^{T} \sum_{j_{1}=0}^{\infty} C_{j_{1}}\left(t_{1}\right) \phi_{j_{1}}\left(t_{1}\right) d t_{1}-\sum_{j_{1}=0}^{p_{1}} \int_{t}^{T} C_{j_{1}}\left(t_{1}\right) \phi_{j_{1}}\left(t_{1}\right) d t_{1}\right| & \leqslant \int_{t}^{T}\left|\psi_{2}\left(t_{1}\right) G^{p_{1}}\left(t_{1}\right)\right| d t_{1} \\
& \leqslant C \int_{t}^{T}\left|G^{p_{1}}\left(t_{1}\right)\right| d t_{1},
\end{aligned}
$$

where

$$
G^{p}(\tau) \stackrel{\text { def }}{=} \sum_{j=p+1}^{\infty} \int_{t}^{\tau} \psi_{1}(s) \phi_{j}(s) d s \phi_{j}(\tau) .
$$

We consider the case of Legendre polynomials. We have:

$$
\left|G^{p_{1}}\left(t_{1}\right)\right|=\frac{1}{2}\left|\sum_{j_{1}=p_{1}+1}^{\infty}\left(2 j_{1}+1\right) \int_{-1}^{z\left(t_{1}\right)} \psi_{1}(u(y)) P_{j_{1}}(y) d y P_{j_{1}}\left(z\left(t_{1}\right)\right)\right|,
$$

where

$$
u(y)=\frac{T-t}{2} y+\frac{T+t}{2}, \quad z(s)=\left(s-\frac{T+t}{2}\right) \frac{2}{T-t},
$$

and $\left\{P_{j}(s)\right\}_{j=0}^{\infty}$ is a complete orthonormal system of Legendre polynomials in the space $L_{2}([-1,1])$.

Throughout the paper, for a rational $q$ we let

$$
J_{q}(t, T) \stackrel{\text { def }}{=} \int_{t}^{T} \frac{d s}{\left(1-z^{2}(s)\right)^{q}}, \quad I_{q}(x) \stackrel{\text { def }}{=} \int_{-1}^{x} \frac{d y}{\left(1-y^{2}\right)^{q}}, \quad f_{q}(x) \stackrel{\text { def }}{=} \frac{1}{\left(1-z^{2}(x)\right)^{q}} .
$$

It follows from (24) and the formula [12]

$$
P_{j+1}^{\prime}(x)-P_{j-1}^{\prime}(x)=(2 j+1) P_{j}(x), \quad j=1,2, \ldots,
$$

where the prime denotes the derivative with respect to $x$, that

$$
\begin{aligned}
\left|G^{p_{1}}\left(t_{1}\right)\right|= & \frac{1}{2} \mid \sum_{j_{1}=p_{1}+1}^{\infty}\left(\left(P_{j_{1}+1}\left(z\left(t_{1}\right)\right)-P_{j_{1}-1}\left(z\left(t_{1}\right)\right)\right) \psi_{1}\left(t_{1}\right)\right. \\
& \left.-\frac{T-t}{2} \int_{-1}^{z\left(t_{1}\right)}\left(P_{j_{1}+1}(y)-P_{j_{1}-1}(y)\right) \psi_{1}^{\prime}(u(y)) d y\right) P_{j_{1}}\left(z\left(t_{1}\right)\right) \mid \\
\leqslant & C_{0}\left|\sum_{j_{1}=p_{1}+1}^{\infty}\left(P_{j_{1}+1}\left(z\left(t_{1}\right)\right) P_{j_{1}}\left(z\left(t_{1}\right)\right)-P_{j_{1}-1}\left(z\left(t_{1}\right)\right) P_{j_{1}}\left(z\left(t_{1}\right)\right)\right)\right| \\
& +\frac{T-t}{4} \mid \sum_{j_{1}=p_{1}+1}^{\infty}\left(\psi _ { 1 } ^ { \prime } ( t _ { 1 } ) \left(\frac{P_{j_{1}+2}\left(z\left(t_{1}\right)\right)-P_{j_{1}}\left(z\left(t_{1}\right)\right)}{2 j_{1}+3}\right.\right. \\
& \left.-\frac{P_{j_{1}}\left(z\left(t_{1}\right)\right)-P_{j_{1}-2}\left(z\left(t_{1}\right)\right)}{2 j_{1}-1}\right)-\frac{T-t}{2} \int_{-1}^{z\left(t_{1}\right)}\left(\frac{P_{j_{1}+2}(y)-P_{j_{1}}(y)}{2 j_{1}+3}\right. \\
& \left.\left.-\frac{P_{j_{1}}(y)-P_{j_{1}-2}(y)}{2 j_{1}-1}\right) \psi_{1}^{\prime \prime}(u(y)) d y\right) P_{j_{1}}\left(z\left(t_{1}\right)\right) \mid,
\end{aligned}
$$


where $\psi_{1}^{\prime}, \psi_{1}^{\prime \prime}$ are the derivatives of the function $\psi_{1}(s)$ with respect to the variable $u(y)$.

By (27) and the estimate for the Legendre polynomials [12]

$$
\left|P_{n}(y)\right|<\frac{K}{\sqrt{n+1}\left(1-y^{2}\right)^{1 / 4}}, \quad y \in(-1,1), \quad n \in \mathbb{N},
$$

for $t_{1} \in(t, T)$ we obtain:

$$
\begin{aligned}
\left|G^{p_{1}}\left(t_{1}\right)\right|< & C_{0}\left|\lim _{n \rightarrow \infty} \sum_{j_{1}=p_{1}+1}^{n}\left(P_{j_{1}+1}\left(z\left(t_{1}\right)\right) P_{j_{1}}\left(z\left(t_{1}\right)\right)-P_{j_{1}-1}\left(z\left(t_{1}\right)\right) P_{j_{1}}\left(z\left(t_{1}\right)\right)\right)\right| \\
& +C_{1} \sum_{j_{1}=p_{1}+1}^{\infty} \frac{1}{j_{1}^{2}}\left(f_{\frac{1}{2}}\left(t_{1}\right)+I_{\frac{1}{4}}\left(z\left(t_{1}\right)\right) f_{\frac{1}{4}}\left(t_{1}\right)\right) \\
< & C_{0}\left|\lim _{n \rightarrow \infty}\left(P_{n+1}\left(z\left(t_{1}\right)\right) P_{n}\left(z\left(t_{1}\right)\right)-P_{p_{1}}\left(z\left(t_{1}\right)\right) P_{p_{1}+1}\left(z\left(t_{1}\right)\right)\right)\right| \\
& +C_{1} \sum_{j_{1}=p_{1}+1}^{\infty} \frac{1}{j_{1}^{2}}\left(f_{\frac{1}{2}}\left(t_{1}\right)+C_{2} f_{\frac{1}{4}}\left(t_{1}\right)\right) \\
< & C_{3} \lim _{n \rightarrow \infty}\left(\frac{1}{n}+\frac{1}{p_{1}}\right) f_{\frac{1}{2}}\left(t_{1}\right)+C_{1} \sum_{j_{1}=p_{1}+1}^{\infty} \frac{1}{j_{1}^{2}}\left(f_{\frac{1}{2}}\left(t_{1}\right)+C_{2} f_{\frac{1}{4}}\left(t_{1}\right)\right) \\
\leqslant & C_{4}\left(\left(\frac{1}{p_{1}}+\sum_{j_{1}=p_{1}+1}^{\infty} \frac{1}{j_{1}^{2}}\right) f_{\frac{1}{2}}\left(t_{1}\right)+\sum_{j_{1}=p_{1}+1}^{\infty} \frac{1}{j_{1}^{2}} f_{\frac{1}{4}}\left(t_{1}\right)\right) \leqslant \frac{K}{p_{1}}\left(f_{\frac{1}{2}}\left(t_{1}\right)+f_{\frac{1}{4}}\left(t_{1}\right)\right),
\end{aligned}
$$

where we have employed the inequality:

$$
\sum_{j_{1}=p_{1}+1}^{\infty} \frac{1}{j_{1}^{2}} \leqslant \int_{p_{1}}^{\infty} \frac{d x}{x^{2}}=\frac{1}{p_{1}}
$$

It follows from (23) and $(29)$ that

$\left|\int_{t}^{T} \sum_{j_{1}=0}^{\infty} C_{j_{1}}\left(t_{1}\right) \phi_{j_{1}}\left(t_{1}\right) d t_{1}-\sum_{j_{1}=0}^{p_{1}} \int_{t}^{T} C_{j_{1}}\left(t_{1}\right) \phi_{j_{1}}\left(t_{1}\right) d t_{1}\right|<\frac{K_{1}}{p_{1}}\left(I_{\frac{1}{2}}(1)+I_{\frac{1}{4}}(1)\right) \rightarrow 0 \quad$ as $\quad p_{1} \rightarrow \infty$.

Hence,

$$
\begin{aligned}
\frac{1}{2} \int_{t}^{T} \psi_{1}\left(t_{1}\right) \psi_{2}\left(t_{1}\right) d t_{1} & =\int_{t}^{T} \sum_{j_{1}=0}^{\infty} C_{j_{1}}\left(t_{1}\right) \phi_{j_{1}}\left(t_{1}\right) d t_{1}=\sum_{j_{1}=0}^{\infty} \int_{t}^{T} C_{j_{1}}\left(t_{1}\right) \phi_{j_{1}}\left(t_{1}\right) d t_{1} \\
& =\sum_{j_{1}=0}^{\infty} \int_{t}^{T} \sum_{j_{2}=0}^{\infty} C_{j_{2} j_{1}} \phi_{j_{2}}\left(t_{1}\right) \phi_{j_{1}}\left(t_{1}\right) d t_{1} \\
& =\sum_{j_{1}=0}^{\infty} \sum_{j_{2}=0}^{\infty} \int_{t}^{T} C_{j_{2} j_{1}} \phi_{j_{2}}\left(t_{1}\right) \phi_{j_{1}}\left(t_{1}\right) d t_{1}=\sum_{j_{1}=0}^{\infty} C_{j_{1} j_{1}} .
\end{aligned}
$$

In (31) we have employed the fact that the Fourier-Legendre series

$$
\sum_{j_{2}=0}^{\infty} C_{j_{2} j_{1}} \phi_{j_{2}}\left(t_{1}\right)
$$

of a smooth function $C_{j_{1}}\left(t_{1}\right)$ converges uniformly to this function on each segment $[t+\varepsilon, T-\varepsilon]$ for all $\varepsilon>0$, converges to this function at each point $(t, T)$ and converges to $C_{j_{1}}(t+0)$ and 
$C_{j_{1}}(T-0)$ as $t_{1}=t$ and $t_{1}=T$, respectively [12], [14]. This completes the proof of relation (16) for the Legendre polynomials.

Let $\left\{\phi_{j}(x)\right\}_{j=0}^{\infty}$ be a complete orthonormal system of trigonometric functions in the space $L_{2}([t, T])$. We have:

$$
\begin{aligned}
& \left|\int_{t}^{T} \sum_{j_{1}=0}^{\infty} C_{j_{1}}\left(t_{1}\right) \phi_{j_{1}}\left(t_{1}\right) d t_{1}-\sum_{j_{1}=0}^{p_{1}} \int_{t}^{T} C_{j_{1}}\left(t_{1}\right) \phi_{j_{1}}\left(t_{1}\right) d t_{1}\right| \\
& =\left|\int_{t}^{T} \sum_{j_{1}=p_{1}+1}^{\infty} \psi_{2}\left(t_{1}\right) \phi_{j_{1}}\left(t_{1}\right) \int_{t}^{t_{1}} \psi_{1}(\theta) \phi_{j_{1}}(\theta) d \theta d t_{1}\right| \\
& =\frac{2}{T-t} \mid \int_{t}^{T} \psi_{2}\left(t_{1}\right) \sum_{j_{1}=p_{1}+1}^{\infty}\left(\int_{t}^{t_{1}} \psi_{1}(s) \sin \frac{2 \pi j_{1}(s-t)}{T-t} d s \sin \frac{2 \pi j_{1}\left(t_{1}-t\right)}{T-t}\right. \\
& \left.+\int_{t}^{t_{1}} \psi_{1}(s) \cos \frac{2 \pi j_{1}(s-t)}{T-t} d s \cos \frac{2 \pi j_{1}\left(t_{1}-t\right)}{T-t}\right) d t_{1} \mid \\
& =\frac{1}{\pi} \mid \int_{t}^{T}\left(\psi_{1}(t) \psi_{2}\left(t_{1}\right) \sum_{j_{1}=p_{1}+1}^{\infty} \frac{1}{j_{1}} \sin \frac{2 \pi j_{1}\left(t_{1}-t\right)}{T-t}\right. \\
& +\frac{T-t}{2 \pi} \psi_{2}\left(t_{1}\right) \sum_{j_{1}=p_{1}+1}^{\infty} \frac{1}{j_{1}^{2}}\left(\psi_{1}^{\prime}\left(t_{1}\right)-\psi_{1}^{\prime}(t) \cos \frac{2 \pi j_{1}\left(t_{1}-t\right)}{T-t}\right. \\
& -\int_{t}^{t_{1}} \sin \frac{2 \pi j_{1}(s-t)}{T-t} \psi_{1}^{\prime \prime}(s) d s \sin \frac{2 \pi j_{1}\left(t_{1}-t\right)}{T-t} \\
& \left.\left.-\int_{t}^{t_{1}} \cos \frac{2 \pi j_{1}(s-t)}{T-t} \psi_{1}^{\prime \prime}(s) d s \cos \frac{2 \pi j_{1}\left(t_{1}-t\right)}{T-t}\right)\right) d t_{1} \mid \\
& \leqslant C_{1}\left|\int_{t}^{T} \psi_{2}\left(t_{1}\right) \sum_{j_{1}=p_{1}+1}^{\infty} \frac{1}{j_{1}} \sin \frac{2 \pi j_{1}\left(t_{1}-t\right)}{T-t} d t_{1}\right|+\frac{C_{2}}{p_{1}} \\
& =C_{1}\left|\sum_{j_{1}=p_{1}+1}^{\infty} \frac{1}{j_{1}} \int_{t}^{T} \psi_{2}\left(t_{1}\right) \sin \frac{2 \pi j_{1}\left(t_{1}-t\right)}{T-t} d t_{1}\right|+\frac{C_{2}}{p_{1}},
\end{aligned}
$$

where the last step is implied by the uniform convergence of the series $\sum_{j_{1}=1}^{\infty} \frac{1}{j_{1}} \sin \frac{2 \pi j_{1}\left(t_{1}-t\right)}{T-t}$ by Dirichlet-Abel test [13].

By (32) we get:

$$
\begin{aligned}
& \left|\int_{t}^{T} \sum_{j_{1}=p_{1}+1}^{\infty} \psi_{2}\left(t_{1}\right) \phi_{j_{1}}\left(t_{1}\right) \int_{t}^{t_{1}} \psi_{1}(\theta) \phi_{j_{1}}(\theta) d \theta d t_{1}\right| \\
& \quad \leqslant C_{3}\left|\sum_{j_{1}=p_{1}+1}^{\infty} \frac{1}{j_{1}^{2}}\left(\psi_{2}(T)-\psi_{2}(t)-\int_{t}^{T} \cos \frac{2 \pi j_{1}(s-t)}{T-t} \psi_{2}^{\prime}(s) d s\right)\right|+\frac{C_{2}}{p_{1}} \leqslant \frac{K}{p_{1}} .
\end{aligned}
$$


The further consideration of this case is similar to the proof of relation (16) for the Legendre polynomials. The proof of Theorem 2 is complete.

\section{Proof of Theorem 3}

First we consider the case of Legendre polynomials. It follows from formula (11) with $p_{1}=$ $p_{2}=p_{3}=p$ and standard relations between stochastic Itô and Stratonovich integrals [2] that Theorem 3 is true provided

$$
\begin{aligned}
& \underset{p \rightarrow \infty}{\lim .} \sum_{j_{1}=0}^{p} \sum_{j_{3}=0}^{p} C_{j_{3} j_{1} j_{1}} \zeta_{j_{3}}^{\left(i_{3}\right)}=\frac{1}{2} \int_{t}^{T} \psi_{3}(s) \int_{t}^{s} \psi_{2}\left(s_{1}\right) \psi_{1}\left(s_{1}\right) d s_{1} d \mathbf{f}_{s}^{\left(i_{3}\right)}, \\
& \underset{p \rightarrow \infty}{\operatorname{li} . \mathrm{m} .} \sum_{j_{1}=0}^{p} \sum_{j_{3}=0}^{p} C_{j_{3} j_{3} j_{1}} \zeta_{j_{1}}^{\left(i_{1}\right)}=\frac{1}{2} \int_{t}^{T} \psi_{3}(s) \psi_{2}(s) \int_{t}^{s} \psi_{1}\left(s_{1}\right) d \mathbf{f}_{s_{1}}^{\left(i_{1}\right)} d s, \\
& \underset{p \rightarrow \infty}{\operatorname{li} . \mathrm{m} .} \sum_{j_{1}=0}^{p} \sum_{j_{3}=0}^{p} C_{j_{1} j_{3} j_{1}} \zeta_{j_{3}}^{\left(i_{2}\right)}=0 .
\end{aligned}
$$

Let us prove (34). By Theorem 1 for $k=1$, see also (9), we get:

$$
\frac{1}{2} \int_{t}^{T} \psi_{3}(s) \int_{t}^{s} \psi_{2}\left(s_{1}\right) \psi_{1}\left(s_{1}\right) d s_{1} d \mathbf{f}_{s}^{\left(i_{3}\right)}=\frac{1}{2} \underset{p \rightarrow \infty}{\lim . \sum_{j_{3}=0}^{p}} \tilde{C}_{j_{3}} \zeta_{j_{3}}^{\left(i_{3}\right)}
$$

where

$$
\tilde{C}_{j_{3}}=\int_{t}^{T} \phi_{j_{3}}(s) \psi_{3}(s) \int_{t}^{s} \psi_{2}\left(s_{1}\right) \psi_{1}\left(s_{1}\right) d s_{1} d s
$$

We have:

$$
\begin{aligned}
E_{p} \stackrel{\text { def }}{=} & \mathrm{M}\left(\left(\sum_{j_{1}=0}^{p} \sum_{j_{3}=0}^{p} C_{j_{3} j_{1} j_{1}} \zeta_{j_{3}}^{\left(i_{3}\right)}-\frac{1}{2} \sum_{j_{3}=0}^{p} \tilde{C}_{j_{3}} \zeta_{j_{3}}^{\left(i_{3}\right)}\right)^{2}\right) \\
= & \mathrm{M}\left(\left(\sum_{j_{3}=0}^{p}\left(\sum_{j_{1}=0}^{p} C_{j_{3} j_{1} j_{1}}-\frac{1}{2} \tilde{C}_{j_{3}}\right) \zeta_{j_{3}}^{\left(i_{3}\right)}\right)^{2}\right)=\sum_{j_{3}=0}^{p}\left(\sum_{j_{1}=0}^{p} C_{j_{3} j_{1} j_{1}}-\frac{1}{2} \tilde{C}_{j_{3}}\right)^{2} \\
= & \sum_{j_{3}=0}^{p}\left(\sum_{j_{1}=0}^{p} \int_{t}^{T} \psi_{3}(s) \phi_{j_{3}}(s) \int_{t}^{s} \psi_{2}\left(s_{1}\right) \phi_{j_{1}}\left(s_{1}\right) \int_{t}^{s} \psi_{1}\left(s_{2}\right) \phi_{j_{1}}\left(s_{2}\right) d s_{2} d s_{1} d s\right. \\
= & \sum_{j_{3}=0}^{p}\left(\int _ { t } ^ { T } \psi _ { 3 } ( s ) \phi _ { j _ { 3 } } ( s ) \int _ { t } ^ { s } \left(\sum_{j_{1}=0}^{p} \psi_{2}\left(s_{1}\right) \phi_{j_{1}}\left(s_{1}\right) \int_{t}^{s_{1}} \psi_{1}\left(s_{2}\right) \phi_{j_{1}}\left(s_{2}\right) d s_{2}\right.\right. \\
& \left.\left.-\frac{1}{2} \psi_{1}\left(s_{1}\right) \psi_{2}\left(s_{1}\right)\right) d s_{1} d s\right)^{2} .
\end{aligned}
$$


Letting $t_{1}=t_{2}=s_{1}$ in $(18)$, we obtain that for each $s_{1} \in(t, T)$ :

$$
\sum_{j_{1}=0}^{\infty} \psi_{2}\left(s_{1}\right) \phi_{j_{1}}\left(s_{1}\right) \int_{t}^{s_{1}} \psi_{1}\left(s_{2}\right) \phi_{j_{1}}\left(s_{2}\right) d s_{2}=\frac{1}{2} \psi_{1}\left(s_{1}\right) \psi_{2}\left(s_{1}\right) .
$$

It follows from (37) and (38) that

$$
E_{p}=\sum_{j_{3}=0}^{p}\left(\int_{t}^{T} \psi_{3}(s) \phi_{j_{3}}(s) \int_{t}^{s} \sum_{j_{1}=p+1}^{\infty} \psi_{2}\left(s_{1}\right) \phi_{j_{1}}\left(s_{1}\right) \int_{t}^{s_{1}} \psi_{1}\left(s_{2}\right) \phi_{j_{1}}\left(s_{2}\right) d s_{2} d s_{1} d s\right)^{2} .
$$

By $(39)$ and $(29)$ we obtain

$$
\begin{aligned}
E_{p} & <C_{1} \sum_{j_{3}=0}^{p}\left(\int_{t}^{T}\left|\phi_{j_{3}}(s)\right| \frac{1}{p}\left(I_{\frac{1}{2}}(z(s))+I_{\frac{1}{4}}(z(s))\right) d s\right)^{2}<\frac{C_{2}}{p^{2}} \sum_{j_{3}=0}^{p}\left(\int_{t}^{T}\left|\phi_{j_{3}}(s)\right| d s\right)^{2} \\
& \leqslant \frac{C_{2}(T-t)}{p^{2}} \sum_{j_{3}=0}^{p} \int_{t}^{T} \phi_{j_{3}}^{2}(s) d s=\frac{C_{3} p}{p^{2}} \rightarrow 0
\end{aligned}
$$

as $p \rightarrow \infty$. This completes the proof of (34).

We proceed to proving (35). By Itô formula we find

$$
\frac{1}{2} \int_{t}^{T} \psi_{3}(s) \psi_{2}(s) \int_{t}^{s} \psi_{1}\left(s_{1}\right) d \mathbf{f}_{s_{1}}^{\left(i_{1}\right)} d s=\frac{1}{2} \int_{t}^{T} \psi_{1}\left(s_{1}\right) \int_{s_{1}}^{T} \psi_{3}(s) \psi_{2}(s) d s d \mathbf{f}_{s_{1}}^{\left(i_{1}\right)}
$$

with probability 1 .

Applying Theorem 1 with $k=1$ and (9), we get:

$$
\frac{1}{2} \int_{t}^{T} \psi_{1}(s) \int_{s}^{T} \psi_{3}\left(s_{1}\right) \psi_{2}\left(s_{1}\right) d s_{1} d \mathbf{f}_{s}^{\left(i_{1}\right)}=\frac{1}{2} \underset{p \rightarrow \infty}{\lim . m} \sum_{j_{1}=0}^{p} C_{j_{1}}^{*} \zeta_{j_{1}}^{\left(i_{1}\right)},
$$

where

$$
C_{j_{1}}^{*}=\int_{t}^{T} \psi_{1}(s) \phi_{j_{1}}(s) \int_{s}^{T} \psi_{3}\left(s_{1}\right) \psi_{2}\left(s_{1}\right) d s_{1} d s
$$

We have

$$
\begin{aligned}
E_{p}^{\prime} \stackrel{\text { def }}{=} \mathrm{M}\left(\left(\sum_{j_{1}=0}^{p} \sum_{j_{3}=0}^{p} C_{j_{3} j_{3} j_{1}} \zeta_{j_{1}}^{\left(i_{1}\right)}-\frac{1}{2} \sum_{j_{1}=0}^{p} C_{j_{1}}^{*} \zeta_{j_{1}}^{\left(i_{1}\right)}\right)^{2}\right)= \\
=\mathrm{M}\left(\left(\sum_{j_{1}=0}^{p}\left(\sum_{j_{3}=0}^{p} C_{j_{3} j_{3} j_{1}}-\frac{1}{2} C_{j_{1}}^{*}\right) \zeta_{j_{1}}^{\left(i_{1}\right)}\right)^{2}\right)=\sum_{j_{1}=0}^{p}\left(\sum_{j_{3}=0}^{p} C_{j_{3} j_{3} j_{1}}-\frac{1}{2} C_{j_{1}}^{*}\right)^{2}, \\
C_{j_{3} j_{3} j_{1}}=\int_{t}^{T} \psi_{3}(s) \phi_{j_{3}}(s) \int_{t}^{s} \psi_{2}\left(s_{1}\right) \phi_{j_{3}}\left(s_{1}\right) \int_{t}^{s_{1}} \psi_{1}\left(s_{2}\right) \phi_{j_{1}}\left(s_{2}\right) d s_{2} d s_{1} d s \\
=\int_{t}^{T} \psi_{1}\left(s_{2}\right) \phi_{j_{1}}\left(s_{2}\right) \int_{s_{2}}^{T} \psi_{2}\left(s_{1}\right) \phi_{j_{3}}\left(s_{1}\right) \int_{s_{1}}^{T} \psi_{3}(s) \phi_{j_{3}}(s) d s d s_{1} d s_{2} .
\end{aligned}
$$


By (40)-(42) we find:

$$
\begin{aligned}
E_{p}^{\prime}= & \sum_{j_{1}=0}^{p}\left(\int _ { t } ^ { T } \psi _ { 1 } ( s _ { 2 } ) \phi _ { j _ { 1 } } ( s _ { 2 } ) \int _ { s _ { 2 } } ^ { T } \left(\sum_{j_{3}=0}^{p} \psi_{2}\left(s_{1}\right) \phi_{j_{3}}\left(s_{1}\right) \int_{s_{1}}^{T} \psi_{3}(s) \phi_{j_{3}}(s) d s\right.\right. \\
& \left.\left.-\frac{1}{2} \psi_{3}\left(s_{1}\right) \psi_{2}\left(s_{1}\right)\right) d s_{1} d s_{2}\right)^{2} .
\end{aligned}
$$

Let us show that for all $s_{1} \in(t, T)$, the identity holds:

$$
\sum_{j_{3}=0}^{\infty} \psi_{2}\left(s_{1}\right) \phi_{j_{3}}\left(s_{1}\right) \int_{s_{1}}^{T} \psi_{3}(s) \phi_{j_{3}}(s) d s=\frac{1}{2} \psi_{2}\left(s_{1}\right) \psi_{3}\left(s_{1}\right) .
$$

We denote

$$
K_{1}^{*}\left(t_{1}, t_{2}\right)=\psi_{2}\left(t_{1}\right) \psi_{3}\left(t_{2}\right) \mathbf{1}_{\left\{t_{1}<t_{2}\right\}}+\frac{1}{2} \mathbf{1}_{\left\{t_{1}=t_{2}\right\}} \psi_{2}\left(t_{1}\right) \psi_{3}\left(t_{1}\right), \quad t_{1}, t_{2} \in[t, T] .
$$

We expand the function $K_{1}^{*}\left(t_{1}, t_{2}\right)$ into the Fourier-Legendre series in the interval $(t, T)$ with respect to the variable $t_{2}$ assuming that $t_{1}$ is fixed:

$$
K_{1}^{*}\left(t_{1}, t_{2}\right)=\sum_{j_{3}=0}^{\infty} \psi_{2}\left(t_{1}\right) \int_{t_{1}}^{T} \psi_{3}(s) \phi_{j_{3}}(s) d s \phi_{j_{3}}\left(t_{2}\right), \quad\left(t_{2} \neq t, \quad t_{2} \neq T .\right.
$$

Letting $t_{1}=t_{2}=s_{1}$ in (45), we obtain (44), see also the proof of formula (38).

By (43) and (44) we obtain:

$$
E_{p}^{\prime}=\sum_{j_{1}=0}^{p}\left(\int_{t}^{T} \psi_{1}\left(s_{2}\right) \phi_{j_{1}}\left(s_{2}\right) \int_{s_{2}}^{T} \sum_{j_{3}=p+1}^{\infty} \psi_{2}\left(s_{1}\right) \phi_{j_{3}}\left(s_{1}\right) \int_{s_{1}}^{T} \psi_{3}(s) \phi_{j_{3}}(s) d s d s_{1} d s_{2}\right)^{2} .
$$

Arguing as in the proof of estimate 29 , for a twice continuously differentiable function $\psi_{3}(s)$ we obtain the following inequality:

$$
\left|\sum_{j_{3}=p+1}^{\infty} \phi_{j_{3}}\left(s_{1}\right) \int_{s_{1}}^{T} \psi_{3}(s) \phi_{j_{3}}(s) d s\right|<\frac{C}{p}\left(f_{\frac{1}{2}}\left(s_{1}\right)+f_{\frac{1}{4}}\left(s_{1}\right)\right),
$$

where $s_{1} \in(t, T)$. The rest of the proof of (35) is similar to the proof of (34).

We proceed to proving (36). We have

$$
\begin{aligned}
E_{p}^{\prime \prime} \stackrel{\text { def }}{=} & \mathrm{M}\left(\left(\sum_{j_{1}=0}^{p} \sum_{j_{3}=0}^{p} C_{j_{1} j_{3} j_{1}} \zeta_{j_{3}}^{\left(i_{2}\right)}\right)^{2}\right)=\sum_{j_{3}=0}^{p}\left(\sum_{j_{1}=0}^{p} C_{j_{1} j_{3} j_{1}}\right)^{2}, \\
C_{j_{1} j_{3} j_{1}} & =\int_{t}^{T} \psi_{3}(s) \phi_{j_{1}}(s) \int_{t}^{s} \psi_{2}\left(s_{1}\right) \phi_{j_{3}}\left(s_{1}\right) \int_{t}^{s_{1}} \psi_{1}\left(s_{2}\right) \phi_{j_{1}}\left(s_{2}\right) d s_{2} d s_{1} d s \\
& =\int_{t}^{T} \psi_{2}\left(s_{1}\right) \phi_{j_{3}}\left(s_{1}\right) \int_{t}^{s_{1}} \psi_{1}\left(s_{2}\right) \phi_{j_{1}}\left(s_{2}\right) d s_{2} \int_{s_{1}}^{T} \psi_{3}(s) \phi_{j_{1}}(s) d s d s_{1} .
\end{aligned}
$$

We substitute 49) into 48):

$$
E_{p}^{\prime \prime}=\sum_{j_{3}=0}^{p}\left(\int_{t}^{T} \psi_{2}\left(s_{1}\right) \phi_{j_{3}}\left(s_{1}\right) \sum_{j_{1}=0}^{p} \int_{t}^{s_{1}} \psi_{1}(\theta) \phi_{j_{1}}(\theta) d \theta \int_{s_{1}}^{T} \psi_{3}(s) \phi_{j_{1}}(s) d s d s_{1}\right)^{2} .
$$


Let $\tilde{K}\left(t_{1}, t_{2}\right)=\psi_{1}\left(t_{1}\right) \mathbf{1}_{\left\{t_{1}<t_{2}\right\}}, t_{1}, t_{2} \in[t, T]$. We expand the function $\tilde{K}\left(t_{1}, t_{2}\right)$ into the FourierLegendre series on the interval $(t, T)$ with respect to the variable $t_{1}$ for a fixed $t_{2}$ :

$$
\tilde{K}\left(t_{1}, t_{2}\right)=\sum_{j_{1}=0}^{\infty} \int_{t}^{t_{2}} \psi_{1}(s) \phi_{j_{1}}(s) d s \phi_{j_{1}}\left(t_{1}\right), \quad t_{1} \neq t_{2} .
$$

Employing (51), we obtain:

$$
\begin{aligned}
& \sum_{j_{1}=0}^{p} \int_{t}^{s_{1}} \psi_{1}(\theta) \phi_{j_{1}}(\theta) d \theta \int_{s_{1}}^{T} \psi_{3}(s) \phi_{j_{1}}(s) d s=\int_{s_{1}}^{T} \psi_{3}(s)\left(\sum_{j_{1}=0}^{p} \phi_{j_{1}}(s) \int_{t}^{s_{1}} \psi_{1}(\theta) \phi_{j_{1}}(\theta) d \theta\right) d s \\
& \quad=\int_{s_{1}}^{T} \psi_{3}(s)\left(\sum_{j_{1}=0}^{\infty} \phi_{j_{1}}(s) \int_{t}^{s_{1}} \psi_{1}(\theta) \phi_{j_{1}}(\theta) d \theta-\sum_{j_{1}=p+1}^{\infty} \phi_{j_{1}}(s) \int_{t}^{s_{1}} \psi_{1}(\theta) \phi_{j_{1}}(\theta) d \theta\right) d s \\
& \quad=\int_{s_{1}}^{T} \psi_{3}(s) \psi_{1}(s) \mathbf{1}_{\left\{s<s_{1}\right\}} d s-\int_{s_{1}}^{T} \psi_{3}(s) \sum_{j_{1}=p+1}^{\infty} \phi_{j_{1}}(s) \int_{t}^{s_{1}} \psi_{1}(\theta) \phi_{j_{1}}(\theta) d \theta d s \\
& \quad=-\int_{s_{1}}^{T} \psi_{3}(s) \sum_{j_{1}=p+1}^{\infty} \phi_{j_{1}}(s) \int_{t}^{s_{1}} \psi_{1}(\theta) \phi_{j_{1}}(\theta) d \theta d s .
\end{aligned}
$$

We substitute (52) into $(50)$ :

$$
\begin{aligned}
E_{p}^{\prime \prime} & =\sum_{j_{3}=0}^{p}\left(\int_{t}^{T} \psi_{2}\left(s_{1}\right) \phi_{j_{3}}\left(s_{1}\right) \int_{s_{1}}^{T} \psi_{3}(s) \sum_{j_{1}=p+1}^{\infty} \phi_{j_{1}}(s) \int_{t}^{s_{1}} \psi_{1}(\theta) \phi_{j_{1}}(\theta) d \theta d s d s_{1}\right)^{2} \\
& =\sum_{j_{3}=0}^{p}\left(\lim _{N \rightarrow \infty} \sum_{l=0}^{N-1} \psi_{2}\left(u_{l}^{*}\right) \phi_{j_{3}}\left(u_{l}^{*}\right) \int_{u_{l}^{*}}^{T} \psi_{3}(s) \sum_{j_{1}=p+1}^{\infty} \phi_{j_{1}}(s) \int_{t}^{u_{l}^{*}} \psi_{1}(\theta) \phi_{j_{1}}(\theta) d \theta d s \Delta u_{l}\right)^{2} \\
& =\sum_{j_{3}=0}^{p}\left(\lim _{N \rightarrow \infty} \sum_{l=0}^{N-1} \psi_{2}\left(u_{l}^{*}\right) \phi_{j_{3}}\left(u_{l}^{*}\right) \sum_{j_{1}=p+1}^{\infty} \int_{u_{l}^{*}}^{T} \psi_{3}(s) \phi_{j_{1}}(s) d s \int_{t}^{u_{l}^{*}} \psi_{1}(\theta) \phi_{j_{1}}(\theta) d \theta \Delta u_{l}\right)^{2},
\end{aligned}
$$

where

$$
t=u_{0}<u_{1}<\ldots<u_{N}=T, \quad \Delta u_{l}=u_{l+1}-u_{l}
$$

$u_{l}^{*}$ is the point of the minimum of the function $\left(1-(z(s))^{2}\right)^{-\alpha}(0<\alpha<1)$ in the interval $\left[u_{l}, u_{l+1}\right]$, and

$$
\max _{0 \leqslant l \leqslant N-1} \Delta u_{l} \rightarrow 0 \quad \text { as } \quad N \rightarrow \infty, \quad l=0,1, \ldots, N-1 .
$$

The last step in (53) is made on the base of the uniform convergence of the Fourier-Legendre series for the function $\tilde{K}\left(s, u_{l}^{*}\right)$ on the segment $\left[u_{l}^{*}+\varepsilon, T-\varepsilon\right]$ for all $\varepsilon>0$ since $\tilde{K}\left(s, u_{l}^{*}\right) \equiv 0$ as $s \in\left[u_{l}^{*}, T\right][12],[14]$. 
We have

$$
\begin{aligned}
\int_{t}^{x} \psi_{1}(s) \phi_{j_{1}}(s) d s= & \frac{\sqrt{(T-t)\left(2 j_{1}+1\right)}}{2} \int_{-1}^{z(x)} P_{j_{1}}(y) \psi(u(y)) d y \\
= & \frac{\sqrt{T-t}}{2 \sqrt{2 j_{1}+1}}\left(\left(P_{j_{1}+1}(z(x))-P_{j_{1}-1}(z(x))\right) \psi_{1}(x)\right. \\
& -\frac{T-t}{2} \int_{-1}^{z(x)}\left(\left(P_{j_{1}+1}(y)-P_{j_{1}-1}(y)\right) \psi_{1}^{\prime}(u(y)) d y\right),
\end{aligned}
$$

where $x \in(t, T), j_{1} \geqslant p+1, z(x)$ and $u(y)$ are given by identities (25), $\psi_{1}{ }^{\prime}$ is the derivative of the function $\psi_{1}(s)$ with respect to the variable $u(y)$.

We note that in (54) we have employed the well-known property of the Legendre polynomials: $P_{j+1}(-1)=-P_{j}(-1), j=0,1,2, \ldots$ and $(26)$.

By (28) and (54) we find:

$$
\left|\int_{t}^{x} \psi_{1}(s) \phi_{j_{1}}(s) d s\right|<\frac{C}{j_{1}}\left(f_{\frac{1}{4}}(x)+C_{1}\right), \quad x \in(t, T) .
$$

Similar to (55) and in view of the identities $P_{j}(1)=1, j=0,1,2, \ldots$, for the integral similar to one in the left hand side of (55) but with the integration limits $x$ and $T$ we obtain an estimate of form (55). Combining estimate (55) and its analogue for the integral with the integration limits $x$ and $T$, we get:

$$
\left|\int_{t}^{x} \psi_{1}(s) \phi_{j_{1}}(s) d s \int_{x}^{T} \psi_{3}(s) \phi_{j_{1}}(s) d s\right|<\frac{K}{j_{1}^{2}}\left(f_{\frac{1}{2}}(x)+K_{1}\right), \quad x \in(t, T) .
$$

We estimate the right hand side in (53) by employing (56):

$$
\begin{aligned}
E_{p}^{\prime \prime} & \leqslant C \sum_{j_{3}=0}^{p}\left(\lim _{N \rightarrow \infty} \sum_{l=0}^{N-1}\left|\phi_{j_{3}}\left(u_{l}^{*}\right)\right| \sum_{j_{1}=p+1}^{\infty} \frac{1}{j_{1}^{2}}\left(f_{\frac{1}{2}}\left(u_{l}^{*}\right)+K_{1}\right) \Delta u_{l}\right)^{2} \\
& \leqslant \frac{C_{1}}{p^{2}} \sum_{j_{3}=0}^{p}\left(\lim _{N \rightarrow \infty} \sum_{l=0}^{N-1}\left(f_{\frac{3}{4}}\left(u_{l}^{*}\right)+K_{1} f_{\frac{1}{4}}\left(u_{l}^{*}\right)\right) \Delta u_{l}\right)^{2} \\
& \leqslant \frac{C_{1}}{p^{2}} \sum_{j_{3}=0}^{p}\left(\lim _{N \rightarrow \infty}\left(J_{\frac{3}{4}}(t, T)+K_{1} J_{\frac{1}{4}}(t, T)\right)\right)^{2}=\frac{C_{1}}{p^{2}} \sum_{j_{3}=0}^{p}\left(J_{\frac{3}{4}}(t, T)+K_{1} J_{\frac{1}{4}}(t, T)\right)^{2} \\
& =\frac{C_{1}(T-t)^{2} p}{4 p^{2}}\left(I_{\frac{3}{4}}(1)+K_{1} I_{\frac{1}{4}}(1)\right)^{2} \leqslant \frac{C_{2}}{p} \rightarrow 0 \text { as } p \rightarrow \infty .
\end{aligned}
$$

This proves (36) and this completes the proof of Theorem 3 for the Legendre polynomials.

We proceed to proving Theorem 3 for trigonometric functions. Similar to inequality (33) we obtain:

$$
\left|\int_{s_{2}}^{T} \sum_{j_{3}=p+1}^{\infty} \psi_{2}\left(s_{1}\right) \phi_{j_{3}}\left(s_{1}\right) \int_{s_{1}}^{T} \psi_{3}(s) \phi_{j_{3}}(s) d s d s_{1}\right| \leqslant \frac{K_{1}}{p}
$$


where $s_{2}$ is fixed. Employing (33) and (39), we obtain:

$$
\begin{aligned}
E_{p} & \leqslant K \sum_{j_{3}=0}^{p}\left(\int\left|\int_{t}^{T} \sum_{t}^{s} \sum_{j_{1}=p+1}^{\infty} \psi_{2}\left(s_{1}\right) \phi_{j_{1}}\left(s_{1}\right) \int_{t}^{s_{1}} \psi_{1}\left(s_{2}\right) \phi_{j_{1}}\left(s_{2}\right) d s_{2} d s_{1}\right| d s\right)^{2} \\
& =K \sum_{j_{3}=0}^{p}\left(\lim _{N \rightarrow \infty} \sum_{l=0}^{N-1}\left|\int_{t}^{u_{l}^{*}} \sum_{j_{1}=p+1}^{\infty} \psi_{2}\left(s_{1}\right) \phi_{j_{1}}\left(s_{1}\right) \int_{t}^{s_{1}} \psi_{1}\left(s_{2}\right) \phi_{j_{1}}\left(s_{2}\right) d s_{2} d s_{1}\right| \Delta u_{l}\right)^{2} \\
& \leqslant K \sum_{j_{3}=0}^{p}\left(\lim _{N \rightarrow \infty} \sum_{l=0}^{N-1} \frac{K_{1}}{p} \Delta u_{l}\right)^{2} \leqslant \frac{K_{2}}{p^{2}} \sum_{j_{3}=0}^{p}(T-t)^{2} \leqslant \frac{C}{p} \rightarrow 0 \quad \text { as } \quad p \rightarrow \infty,
\end{aligned}
$$

where

$$
\begin{array}{r}
t=u_{0}<u_{1}<\ldots<u_{N}=T, \quad \Delta u_{l}=u_{l+1}-u_{l}, \quad u_{l}^{*} \in\left[u_{l}, u_{l+1}\right], \\
\max _{0 \leqslant l \leqslant N-1} \Delta u_{l} \rightarrow 0 \quad \text { as } \quad N \rightarrow \infty, \quad l=0,1, \ldots, N-1 .
\end{array}
$$

In the same way, employing (58) and (46), we obtain that $E_{p}^{\prime} \rightarrow 0$ as $p \rightarrow \infty$.

It is easy to see that in the considered case, the estimate holds:

$$
\left|\int_{t}^{x} \psi_{1}(s) \phi_{j_{1}}(s) d s \int_{x}^{T} \psi_{3}(s) \phi_{j_{1}}(s) d s\right|<\frac{K}{j_{1}^{2}}, \quad j_{1} \neq 0 .
$$

It follows from $(53)$ and $(60)$ that

$$
\begin{aligned}
E_{p}^{\prime \prime} & \leqslant K_{1} \sum_{j_{3}=0}^{p}\left(\lim _{N \rightarrow \infty} \sum_{l=0}^{N-1} \sum_{j_{1}=p+1}^{\infty}\left|\int_{u_{l}^{*}}^{T} \psi_{3}(s) \phi_{j_{1}}(s) d s \int_{t}^{u_{l}^{*}} \psi_{1}(\theta) \phi_{j_{1}}(\theta) d \theta\right| \Delta u_{l}\right)^{2} \\
& \leqslant K_{2} \sum_{j_{3}=0}^{p}\left(\lim _{N \rightarrow \infty} \sum_{l=0}^{N-1} \sum_{j_{1}=p+1}^{\infty} \frac{1}{j_{1}^{2}} \Delta u_{l}\right)^{2} \leqslant \frac{K_{2}}{p^{2}} \sum_{j_{3}=0}^{p}(T-t)^{2} \leqslant \frac{C}{p} \rightarrow 0 \quad \text { as } \quad p \rightarrow \infty .
\end{aligned}
$$

Here we have employed the same notations as in (59). This completes the proof of Theorem 3.

\section{Proof of Theorem 4}

It follows from $(12)$ that

$$
\begin{aligned}
& \underset{p \rightarrow \infty}{\lim .} \sum_{j_{1}, j_{2}, j_{3}, j_{4}=0}^{p} C_{j_{4} j_{3} j_{2} j_{1}} \zeta_{j_{1}}^{\left(i_{1}\right)} \zeta_{j_{2}}^{\left(i_{2}\right)} \zeta_{j_{3}}^{\left(i_{3}\right)} \zeta_{j_{4}}^{\left(i_{4}\right)}=J\left[\psi^{(4)}\right]_{T, t}+\mathbf{1}_{\left\{i_{1}=i_{2} \neq 0\right\}} A_{1}^{\left(i_{3} i_{4}\right)} \\
& +\mathbf{1}_{\left\{i_{1}=i_{3} \neq 0\right\}} A_{2}^{\left(i_{2} i_{4}\right)}+\mathbf{1}_{\left\{i_{1}=i_{4} \neq 0\right\}} A_{3}^{\left(i_{2} i_{3}\right)} \\
& +\mathbf{1}_{\left\{i_{2}=i_{3} \neq 0\right\}} A_{4}^{\left(i_{1} i_{4}\right)}+\mathbf{1}_{\left\{i_{2}=i_{4} \neq 0\right\}} A_{5}^{\left(i_{1} i_{3}\right)} \\
& +\mathbf{1}_{\left\{i_{3}=i_{4} \neq 0\right\}} A_{6}^{\left(i_{1} i_{2}\right)}-\mathbf{1}_{\left\{i_{1}=i_{2} \neq 0\right\}} \mathbf{1}_{\left\{i_{3}=i_{4} \neq 0\right\}} B_{1} \\
& \text { - } \mathbf{1}_{\left\{i_{1}=i_{3} \neq 0\right\}} \mathbf{1}_{\left\{i_{2}=i_{4} \neq 0\right\}} B_{2} \\
& -\mathbf{1}_{\left\{i_{1}=i_{4} \neq 0\right\}} \mathbf{1}_{\left\{i_{2}=i_{3} \neq 0\right\}} B_{3} \text {, }
\end{aligned}
$$

where $J\left[\psi^{(4)}\right]_{T, t}$ is of form (2) as $\psi_{1}(s), \ldots, \psi_{4}(s) \equiv 1$ and $i_{1}, \ldots, i_{4}=0,1, \ldots, m$,

$$
A_{1}^{\left(i_{3} i_{4}\right)}=\underset{p \rightarrow \infty}{\lim .{ }_{j_{4}, j_{3}, j_{1}=0}^{p}} C_{j_{4} j_{3} j_{1} j_{1}} \zeta_{j_{3}}^{\left(i_{3}\right)} \zeta_{j_{4}}^{\left(i_{4}\right)}
$$




$$
\begin{aligned}
& A_{2}^{\left(i_{2} i_{4}\right)}=\lim _{p \rightarrow \infty} \sum_{j_{4}, j_{3}, j_{2}=0}^{p} C_{j_{4} j_{3} j_{2} j_{3}} \zeta_{j_{2}}^{\left(i_{2}\right)} \zeta_{j_{4}}^{\left(i_{4}\right)}, \\
& A_{3}^{\left(i_{2} i_{3}\right)}=\lim _{p \rightarrow \infty} \sum_{j_{4}, j_{3}, j_{2}=0}^{p} C_{j_{4} j_{3} j_{2} j_{4}} \zeta_{j_{2}}^{\left(i_{2}\right)} \zeta_{j_{3}}^{\left(i_{3}\right)}, \\
& A_{4}^{\left(i_{1} i_{4}\right)}=\lim _{p \rightarrow \infty} \sum_{j_{4}, j_{3}, j_{1}=0}^{p} C_{j_{4} j_{3} j_{3} j_{1}} \zeta_{j_{1}}^{\left(i_{1}\right)} \zeta_{j_{4}}^{\left(i_{4}\right)}, \\
& A_{5}^{\left(i_{1} i_{3}\right)}=\lim _{p \rightarrow \infty} \sum_{j_{4}, j_{3}, j_{1}=0}^{p} C_{j_{4} j_{3} j_{4} j_{1}} \zeta_{j_{1}}^{\left(i_{1}\right)} \zeta_{j_{3}}^{\left(i_{3}\right)}, \\
& A_{1}^{\left(i_{1} i_{2}\right)}=\lim _{p \rightarrow \infty} \sum_{p \rightarrow \infty} \sum_{j_{3}, j_{2}, j_{1}=0}^{p} C_{j_{3} j_{3} j_{2} j_{1}} \zeta_{j_{1}}^{\left(i_{1}\right)} \zeta_{j_{2}}^{\left(i_{2}\right)}, \\
& B_{3}=0 \\
& B_{j_{4} j_{4} j_{1} j_{1}}, \quad B_{2}=\lim _{p \rightarrow \infty} \sum_{j_{4}, j_{3}=0}^{p} C_{j_{4}, j_{3}=0}^{p} C_{j_{3} j_{4} j_{3} j_{4}},
\end{aligned}
$$

Interchanging the integration order in Riemann integrals and employing Theorem 1 for $k=2$, see (10), relation (16), Parseval identity and Itô formula, we obtain:

$$
\begin{aligned}
& A_{1}^{\left(i_{3} i_{4}\right)}=\underset{p \rightarrow \infty}{\operatorname{lin} .{ }_{p}} \sum_{j_{4}, j_{3}, j_{1}=0}^{p} \frac{1}{2} \int_{t}^{T} \phi_{j_{4}}(s) \int_{t}^{s} \phi_{j_{3}}\left(s_{1}\right)\left(\int_{t}^{s_{1}} \phi_{j_{1}}\left(s_{2}\right) d s_{2}\right)^{2} d s_{1} d s \zeta_{j_{3}}^{\left(i_{3}\right)} \zeta_{j_{4}}^{\left(i_{4}\right)} \\
& =\underset{p \rightarrow \infty}{\operatorname{li.m}} \sum_{j_{4}, j_{3}=0}^{p} \frac{1}{2} \int_{t}^{T} \phi_{j_{4}}(s) \int_{t}^{s} \phi_{j_{3}}\left(s_{1}\right) \sum_{j_{1}=0}^{p}\left(\int_{t}^{s_{1}} \phi_{j_{1}}\left(s_{2}\right) d s_{2}\right)^{2} d s_{1} d s \zeta_{j_{3}}^{\left(i_{3}\right)} \zeta_{j_{4}}^{\left(i_{4}\right)} \\
& =\underset{p \rightarrow \infty}{\operatorname{li} . m} \sum_{j_{4}, j_{3}=0}^{p} \frac{1}{2} \int_{t}^{T} \phi_{j_{4}}(s) \int_{t}^{s} \phi_{j_{3}}\left(s_{1}\right)\left(\left(s_{1}-t\right)-\sum_{j_{1}=p+1}^{\infty}\left(\int_{t}^{s_{1}} \phi_{j_{1}}\left(s_{2}\right) d s_{2}\right)^{2}\right) d s_{1} d s \\
& \text { - } \zeta_{j_{3}}^{\left(i_{3}\right)} \zeta_{j_{4}}^{\left(i_{4}\right)} \\
& =\underset{p \rightarrow \infty}{\lim } \sum_{j_{4}, j_{3}=0}^{p} \frac{1}{2} \int_{t}^{T} \phi_{j_{4}}(s) \int_{t}^{s} \phi_{j_{3}}\left(s_{1}\right)\left(s_{1}-t\right) d s_{1} d s \zeta_{j_{3}}^{\left(i_{3}\right)} \zeta_{j_{4}}^{\left(i_{4}\right)}-\Delta_{1}^{\left(i_{3} i_{4}\right)} \\
& =\frac{1}{2} \int_{t}^{T} \int_{t}^{s}\left(s_{1}-t\right) d \mathbf{w}_{s_{1}}^{\left(i_{3}\right)} d \mathbf{w}_{s}^{\left(i_{4}\right)} \\
& +\frac{1}{2} \mathbf{1}_{\left\{i_{3}=i_{4} \neq 0\right\}} \lim _{p \rightarrow \infty} \sum_{j_{3}=0}^{p} \int_{t}^{T} \phi_{j_{3}}(s) \int_{t}^{s} \phi_{j_{3}}\left(s_{1}\right)\left(s_{1}-t\right) d s_{1} d s-\Delta_{1}^{\left(i_{3} i_{4}\right)} \\
& =\frac{1}{2} \int_{t}^{T} \int_{t}^{s} \int_{t}^{s_{1}} d s_{2} d \mathbf{w}_{s_{1}}^{\left(i_{3}\right)} d \mathbf{w}_{s}^{\left(i_{4}\right)}+\frac{1}{4} \mathbf{1}_{\left\{i_{3}=i_{4} \neq 0\right\}} \int_{t}^{T}\left(s_{1}-t\right) d s_{1}-\Delta_{1}^{\left(i_{3} i_{4}\right)}
\end{aligned}
$$


with probability 1 , where

$$
\begin{aligned}
\Delta_{1}^{\left(i_{3} i_{4}\right)} & =\underset{p \rightarrow \infty}{\lim .} \sum_{j_{3}, j_{4}=0}^{p} a_{j_{4} j_{3}}^{p} \zeta_{j_{3}}^{\left(i_{3}\right)} \zeta_{j_{4}}^{\left(i_{4}\right)} \\
a_{j_{4} j_{3}}^{p} & =\frac{1}{2} \int_{t}^{T} \phi_{j_{4}}(s) \int_{t}^{s} \phi_{j_{3}}\left(s_{1}\right) \sum_{j_{1}=p+1}^{\infty}\left(\int_{t}^{s_{1}} \phi_{j_{1}}\left(s_{2}\right) d s_{2}\right)^{2} d s_{1} d s .
\end{aligned}
$$

We consider $A_{2}^{\left(i_{2} i_{4}\right)}$ :

$$
\begin{aligned}
& A_{2}^{\left(i_{2} i_{4}\right)}=\operatorname{l.i.m.~}_{p \rightarrow \infty} \sum_{j_{4}, j_{3}, j_{2}=0}^{p}\left(\frac{1}{2} \int_{t}^{T} \phi_{j_{4}}(s)\left(\int_{t}^{s} \phi_{j_{3}}\left(s_{1}\right) d s_{1}\right)^{2} \int_{t}^{s} \phi_{j_{2}}\left(s_{1}\right) d s_{1} d s\right. \\
&-\frac{1}{2} \int_{t}^{T} \phi_{j_{4}}(s) \int_{t}^{s} \phi_{j_{2}}\left(s_{1}\right)\left(\int_{t}^{s_{1}} \phi_{j_{3}}\left(s_{2}\right) d s_{2}\right)^{2} d s_{1} d s \\
&\left.-\frac{1}{2} \int_{t}^{T} \phi_{j_{4}}(s) \int_{t}^{s} \phi_{j_{2}}\left(s_{3}\right)\left(\int_{s_{3}}^{s} \phi_{j_{3}}\left(s_{1}\right) d s_{1}\right)^{2} d s_{3} d s\right) \zeta_{j_{2}}^{\left(i_{2}\right)} \zeta_{j_{4}}^{\left(i_{4}\right)} \\
&=\operatorname{l.i.m.~}_{p \rightarrow \infty} \sum_{j_{4}, j_{2}=0}^{p}\left(\frac{1}{2} \int_{t}^{T} \phi_{j_{4}}(s)(s-t) \int_{t}^{s} \phi_{j_{2}}\left(s_{1}\right) d s_{1} d s\right. \\
&-\frac{1}{2} \int_{t}^{T} \phi_{j_{4}}(s) \int_{t}^{s} \phi_{j_{2}}\left(s_{1}\right)\left(s_{1}-t\right) d s_{1} d s \\
&\left.-\frac{1}{2} \int_{t}^{T} \phi_{j_{4}}(s) \int_{t}^{s} \phi_{j_{2}}\left(s_{3}\right)\left(s-t+t-s_{3}\right) d s_{3} d s\right) \zeta_{j_{2}}^{\left(i_{2}\right)} \zeta_{j_{4}}^{\left(i_{4}\right)} \\
&-\Delta_{2}^{\left(i_{2} i_{4}\right)}+\Delta_{1}^{\left(i_{2} i_{4}\right)}+\Delta_{3}^{\left(i_{2} i_{4}\right)} \\
&=-\Delta_{2}^{\left(i_{2} i_{4}\right)}+\Delta_{1}^{\left(i_{2} i_{4}\right)}+\Delta_{3}^{\left(i_{2} i_{4}\right)}
\end{aligned}
$$

with probability 1 , where

$$
\begin{aligned}
& \Delta_{2}^{\left(i_{2} i_{4}\right)}=\underset{p \rightarrow \infty}{\operatorname{li} . \mathrm{m}} \sum_{j_{4}, j_{2}=0}^{p} b_{j_{4} j_{2}}^{p} \zeta_{j_{2}}^{\left(i_{2}\right)} \zeta_{j_{4}}^{\left(i_{4}\right)}, \\
& \Delta_{3}^{\left(i_{2} i_{4}\right)}=\underset{p \rightarrow \infty}{\operatorname{li} . \mathrm{m} .} \sum_{j_{4}, j_{2}=0}^{p} c_{j_{4} j_{2}}^{p} \zeta_{j_{2}}^{\left(i_{2}\right)} \zeta_{j_{4}}^{\left(i_{4}\right)}, \\
& b_{j_{4} j_{2}}^{p}=\frac{1}{2} \int_{t}^{T} \phi_{j_{4}}(s) \sum_{j_{3}=p+1}^{\infty}\left(\int_{t}^{s} \phi_{j_{3}}\left(s_{1}\right) d s_{1}\right)^{2} \int_{t}^{s} \phi_{j_{2}}\left(s_{1}\right) d s_{1} d s, \\
& c_{j_{4} j_{2}}^{p}=\frac{1}{2} \int_{t}^{T} \phi_{j_{4}}(s) \int_{t}^{s} \phi_{j_{2}}\left(s_{3}\right) \sum_{j_{3}=p+1}^{\infty}\left(\int_{s_{3}}^{s} \phi_{j_{3}}\left(s_{1}\right) d s_{1}\right)^{2} d s_{3} d s .
\end{aligned}
$$


We consider $A_{5}^{\left(i_{1} i_{3}\right)}$ :

$$
\begin{aligned}
& A_{5}^{\left(i_{1} i_{3}\right)}=\underset{p \rightarrow \infty}{\lim . \mathrm{m}} \sum_{j_{4}, j_{3}, j_{1}=0}^{p} \int_{t}^{T} \phi_{j_{1}}\left(s_{3}\right) \int_{s_{3}}^{T} \phi_{j_{4}}\left(s_{2}\right) \int_{s_{2}}^{T} \phi_{j_{3}}\left(s_{1}\right) \int_{s_{1}}^{T} \phi_{j_{4}}(s) d s d s_{1} d s_{2} d s_{3} \\
& \cdot \zeta_{j_{1}}^{\left(i_{1}\right)} \zeta_{j_{3}}^{\left(i_{3}\right)} \\
& =\underset{p \rightarrow \infty}{\operatorname{li} . \mathrm{m} .} \sum_{j_{4}, j_{3}, j_{1}=0}^{p} \int_{t}^{T} \phi_{j_{1}}\left(s_{3}\right) \int_{s_{3}}^{T} \phi_{j_{4}}(s) \int_{s_{3}}^{s} \phi_{j_{3}}\left(s_{1}\right) \int_{s_{3}}^{s_{1}} \phi_{j_{4}}\left(s_{2}\right) d s_{2} d s_{1} d s d s_{3} \\
& \cdot \zeta_{j_{1}}^{\left(i_{1}\right)} \zeta_{j_{3}}^{\left(i_{3}\right)} \\
& =\underset{p \rightarrow \infty}{\lim .} \sum_{j_{4}, j_{3}, j_{1}=0}^{p}\left(\frac{1}{2} \int_{t}^{T} \phi_{j_{1}}\left(s_{3}\right)\left(\int_{s_{3}}^{T} \phi_{j_{4}}(s) d s\right)^{2} \int_{s_{3}}^{T} \phi_{j_{3}}(s) d s d s_{3}\right. \\
& -\frac{1}{2} \int_{t}^{T} \phi_{j_{1}}\left(s_{3}\right) \int_{s_{3}}^{T} \phi_{j_{3}}(s)\left(\int_{s_{3}}^{s} \phi_{j_{4}}\left(s_{1}\right) d s_{1}\right)^{2} d s d s_{3} \\
& \left.-\frac{1}{2} \int_{t}^{T} \phi_{j_{1}}\left(s_{3}\right) \int_{s_{3}}^{T} \phi_{j_{3}}\left(s_{2}\right)\left(\int_{s_{2}}^{T} \phi_{j_{4}}\left(s_{1}\right) d s_{1}\right)^{2} d s_{2} d s_{3}\right) \\
& \cdot \zeta_{j_{1}}^{\left(i_{1}\right)} \zeta_{j_{3}}^{\left(i_{3}\right)} \\
& =\underset{p \rightarrow \infty}{\lim } \sum_{j_{3}, j_{1}=0}^{p}\left(\frac{1}{2} \int_{t}^{T} \phi_{j_{1}}\left(s_{3}\right)\left(T-s_{3}\right) \int_{s_{3}}^{T} \phi_{j_{3}}(s) d s d s_{3}\right. \\
& -\frac{1}{2} \int_{t}^{T} \phi_{j_{1}}\left(s_{3}\right) \int_{s_{3}}^{T} \phi_{j_{3}}(s)\left(s-s_{3}\right) d s d s_{3} \\
& \left.-\frac{1}{2} \int_{t}^{T} \phi_{j_{1}}\left(s_{3}\right) \int_{s_{3}}^{T} \phi_{j_{3}}\left(s_{2}\right)\left(T-s_{2}\right) d s_{2} d s_{3}\right) \zeta_{j_{1}}^{\left(i_{1}\right)} \zeta_{j_{3}}^{\left(i_{3}\right)} \\
& -\Delta_{4}^{\left(i_{1} i_{3}\right)}+\Delta_{5}^{\left(i_{1} i_{3}\right)}+\Delta_{6}^{\left(i_{1} i_{3}\right)} \\
& =-\Delta_{4}^{\left(i_{1} i_{3}\right)}+\Delta_{5}^{\left(i_{1} i_{3}\right)}+\Delta_{6}^{\left(i_{1} i_{3}\right)}
\end{aligned}
$$

where

$$
\begin{aligned}
& \Delta_{4}^{\left(i_{1} i_{3}\right)}=\lim _{p \rightarrow \infty} \sum_{j_{3}, j_{1}=0}^{p} d_{j_{3} j_{1}}^{p} \zeta_{j_{1}}^{\left(i_{1}\right)} \zeta_{j_{3}}^{\left(i_{3}\right)}, \\
& \Delta_{5}^{\left(i_{1} i_{3}\right)}=\lim _{p \rightarrow \infty} \sum_{j_{3}, j_{1}=0}^{p} e_{j_{3} j_{1}}^{p} \zeta_{j_{1}}^{\left(i_{1}\right)} \zeta_{j_{3}}^{\left(i_{3}\right)}, \\
& d_{j_{3} j_{1}}^{p}=\frac{1}{2} \int_{t}^{T} \phi_{j_{1}}\left(s_{3}\right) \sum_{j_{4}=p+1}^{\infty}\left(\int_{s_{3}}^{T} \phi_{j_{4}}(s) d s\right)^{2} \int_{s_{3}}^{T} \phi_{j_{3}}(s) d s d s_{3},
\end{aligned}
$$




$$
\begin{aligned}
e_{j_{3} j_{1}}^{p}= & \frac{1}{2} \int_{t}^{T} \phi_{j_{1}}\left(s_{3}\right) \int_{s_{3}}^{T} \phi_{j_{3}}(s) \sum_{j_{4}=p+1}^{\infty}\left(\int_{s_{3}}^{s} \phi_{j_{4}}\left(s_{1}\right) d s_{1}\right)^{2} d s d s_{3}, \\
\Delta_{6}^{\left(i_{1} i_{3}\right)} & =\operatorname{li}_{p \rightarrow \infty} . \sum_{j_{3}, j_{1}=0}^{p} f_{j_{3} j_{1}}^{p} \zeta_{j_{1}}^{\left(i_{1}\right)} \zeta_{j_{3}}^{\left(i_{3}\right)}, \\
f_{j_{3} j_{1}}^{p}= & \frac{1}{2} \int_{t}^{T} \phi_{j_{1}}\left(s_{3}\right) \int_{s_{3}}^{T} \phi_{j_{3}}\left(s_{2}\right) \sum_{j_{4}=p+1}^{\infty}\left(\int_{s_{2}}^{T} \phi_{j_{4}}\left(s_{1}\right) d s_{1}\right)^{2} d s_{2} d s_{3} \\
= & \frac{1}{2} \int_{t}^{T} \phi_{j_{3}}\left(s_{2}\right) \sum_{j_{4}=p+1}^{\infty}\left(\int_{s_{2}}^{T} \phi_{j_{4}}\left(s_{1}\right) d s_{1}\right)^{2} \int_{t}^{s_{2}} \phi_{j_{1}}\left(s_{3}\right) d s_{3} d s_{2} .
\end{aligned}
$$

Moreover,

$$
\begin{aligned}
& A_{3}^{\left(i_{2} i_{3}\right)}+A_{5}^{\left(i_{2} i_{3}\right)}=\lim _{p \rightarrow \infty} \sum_{j_{4}, j_{3}, j_{2}=0}^{p}\left(C_{j_{4} j_{3} j_{2} j_{4}}+C_{j_{4} j_{3} j_{4} j_{2}}\right) \zeta_{j_{2}}^{\left(i_{2}\right)} \zeta_{j_{3}}^{\left(i_{3}\right)} \\
& =\underset{p \rightarrow \infty}{\operatorname{li.m}} \sum_{j_{4}, j_{3}, j_{2}=0}^{p} \int_{t}^{T} \phi_{j_{4}}(s) \int_{t}^{s} \phi_{j_{3}}\left(s_{1}\right) \int_{t}^{s_{1}} \phi_{j_{2}}\left(s_{2}\right) \int_{t}^{s_{1}} \phi_{j_{4}}\left(s_{3}\right) d s_{3} d s_{2} d s_{1} d s \\
& \text { - } \zeta_{j_{2}}^{\left(i_{2}\right)} \zeta_{j_{3}}^{\left(i_{3}\right)} \\
& =\underset{p \rightarrow \infty}{\lim .} \sum_{j_{4}, j_{3}, j_{2}=0}^{p} \int_{t}^{T} \phi_{j_{3}}\left(s_{1}\right) \int_{t}^{s_{1}} \phi_{j_{2}}\left(s_{2}\right) \int_{t}^{s_{1}} \phi_{j_{4}}\left(s_{3}\right) d s_{3} d s_{2} \int_{s_{1}}^{T} \phi_{j_{4}}(s) d s d s_{1} \\
& \text { - } \zeta_{j_{2}}^{\left(i_{2}\right)} \zeta_{j_{3}}^{\left(i_{3}\right)} \\
& =\underset{p \rightarrow \infty}{\lim .} \sum_{j_{4}, j_{3}, j_{2}=0}^{p}\left(\int_{t}^{T} \phi_{j_{3}}\left(s_{1}\right) \int_{t}^{s_{1}} \phi_{j_{2}}\left(s_{2}\right) \int_{t}^{T} \phi_{j_{4}}\left(s_{3}\right) d s_{3} \int_{s_{1}}^{T} \phi_{j_{4}}(s) d s d s_{2} d s_{1}\right. \\
& \left.-\int_{t}^{T} \phi_{j_{3}}\left(s_{1}\right) \int_{t}^{s_{1}} \phi_{j_{2}}\left(s_{2}\right)\left(\int_{s_{1}}^{T} \phi_{j_{4}}(s) d s\right)^{2} d s_{2} d s_{1}\right) \zeta_{j_{2}}^{\left(i_{2}\right)} \zeta_{j_{3}}^{\left(i_{3}\right)} \\
& =\underset{p \rightarrow \infty}{\lim . \sum_{j_{3}, j_{2}=0}^{p}} \int_{t}^{T} \phi_{j_{3}}\left(s_{1}\right) \int_{t}^{s_{1}} \phi_{j_{2}}\left(s_{2}\right)\left(\left(T-s_{1}\right)-\sum_{j_{4}=0}^{p}\left(\int_{s_{1}}^{T} \phi_{j_{4}}\left(s_{3}\right) d s_{3}\right)^{2}\right) d s_{2} d s_{1} \\
& =2 \Delta_{6}^{\left(i_{2} i_{3}\right)} \\
& \text { - } \zeta_{j_{2}}^{\left(i_{2}\right)} \zeta_{j_{3}}^{\left(i_{3}\right)}
\end{aligned}
$$

with probability 1 . This is why

$$
\begin{aligned}
A_{3}^{\left(i_{2} i_{3}\right)} & =2 \Delta_{6}^{\left(i_{2} i_{3}\right)}-A_{5}^{\left(i_{2} i_{3}\right)} \\
& =\Delta_{4}^{\left(i_{2} i_{3}\right)}-\Delta_{5}^{\left(i_{2} i_{3}\right)}+\Delta_{6}^{\left(i_{2} i_{3}\right)}
\end{aligned}
$$

with probability 1 . 
We consider $A_{4}^{\left(i_{1} i_{4}\right)}$ :

$$
\begin{aligned}
& A_{4}^{\left(i_{1} i_{4}\right)}=\underset{p \rightarrow \infty}{\operatorname{li} . \mathrm{m} .} \sum_{j_{4}, j_{3}, j_{1}=0}^{p} \int_{t}^{T} \phi_{j_{4}}(s) \int_{t}^{s} \phi_{j_{1}}\left(s_{3}\right) \int_{s_{3}}^{s} \phi_{j_{3}}\left(s_{2}\right) \int_{s_{2}}^{s} \phi_{j_{3}}\left(s_{1}\right) d s_{1} d s_{2} d s_{3} d s \zeta_{j_{1}}^{\left(i_{1}\right)} \zeta_{j_{4}}^{\left(i_{4}\right)} \\
& =\underset{p \rightarrow \infty}{\lim } \sum_{j_{4}, j_{1}=0}^{p} \frac{1}{2} \int_{t}^{T} \phi_{j_{4}}(s) \int_{t}^{s} \phi_{j_{1}}\left(s_{3}\right) \sum_{j_{3}=0}^{p}\left(\int_{s_{3}}^{s} \phi_{j_{3}}\left(s_{2}\right) d s_{2}\right)^{2} d s_{3} d s \zeta_{j_{1}}^{\left(i_{1}\right)} \zeta_{j_{4}}^{\left(i_{4}\right)} \\
& =\underset{p \rightarrow \infty}{\lim } \sum_{j_{4}, j_{1}=0}^{p} \frac{1}{2} \int_{t}^{T} \phi_{j_{4}}(s) \int_{t}^{s} \phi_{j_{1}}\left(s_{3}\right)\left(s-s_{3}\right) d s_{3} d s \zeta_{j_{1}}^{\left(i_{1}\right)} \zeta_{j_{4}}^{\left(i_{4}\right)}-\Delta_{3}^{\left(i_{1} i_{4}\right)} \\
& =\frac{1}{2} \int_{t}^{T} \int_{t}^{s}\left(s-s_{3}\right) d \mathbf{w}_{s_{3}}^{\left(i_{1}\right)} d \mathbf{w}_{s}^{\left(i_{4}\right)}+\frac{1}{2} \mathbf{1}_{\left\{i_{1}=i_{4} \neq 0\right\}} \lim _{p \rightarrow \infty} \sum_{j_{4}=0}^{p} \int_{t}^{T} \phi_{j_{4}}(s) \int_{t}^{s} \phi_{j_{4}}\left(s_{3}\right)\left(s-s_{3}\right) d s_{3} d s-\Delta_{3}^{\left(i_{1} i_{4}\right)} \\
& =\frac{1}{2} \int_{t}^{T} \int_{t}^{s_{2}} \int_{t}^{s_{1}} d \mathbf{w}_{s}^{\left(i_{1}\right)} d s_{1} d \mathbf{w}_{s_{2}}^{\left(i_{4}\right)}+\frac{1}{2} \mathbf{1}_{\left\{i_{1}=i_{4} \neq 0\right\}}\left(\sum_{j_{4}=0}^{\infty} \int_{t}^{T}(s-t) \phi_{j_{4}}(s) \int_{t}^{s} \phi_{j_{4}}\left(s_{3}\right) d s_{3} d s\right. \\
& \left.-\sum_{j_{4}=0}^{\infty} \int_{t}^{T} \phi_{j_{4}}(s) \int_{t}^{s}\left(s_{3}-t\right) \phi_{j_{4}}\left(s_{3}\right) d s_{3} d s\right)-\Delta_{3}^{\left(i_{1} i_{4}\right)}=\frac{1}{2} \int_{t}^{T} \int_{t}^{s_{2}} \int_{t}^{s_{1}} d \mathbf{w}_{s}^{\left(i_{1}\right)} d s_{1} d \mathbf{w}_{s_{2}}^{\left(i_{4}\right)}-\Delta_{3}^{\left(i_{1} i_{4}\right)}
\end{aligned}
$$

with probability 1 . We consider $A_{6}^{\left(i_{1} i_{2}\right)}$ :

$$
\begin{aligned}
& A_{6}^{\left(i_{1} i_{2}\right)}=\underset{p \rightarrow \infty}{\lim . \mathrm{m}} \sum_{j_{3}, j_{2}, j_{1}=0}^{p} \int_{t}^{T} \phi_{j_{1}}\left(s_{3}\right) \int_{s_{3}}^{T} \phi_{j_{2}}\left(s_{2}\right) \int_{s_{2}}^{T} \phi_{j_{3}}\left(s_{1}\right) \int_{s_{1}}^{T} \phi_{j_{3}}(s) d s d s_{1} d s_{2} d s_{3} \zeta_{j_{1}}^{\left(i_{1}\right)} \zeta_{j_{2}}^{\left(i_{2}\right)} \\
& =\underset{p \rightarrow \infty}{\operatorname{li} . \mathrm{m}} \sum_{j_{1}, j_{2}=0}^{p} \frac{1}{2} \int_{t}^{T} \phi_{j_{1}}\left(s_{3}\right) \int_{s_{3}}^{T} \phi_{j_{2}}\left(s_{2}\right) \sum_{j_{3}=0}^{p}\left(\int_{s_{2}}^{T} \phi_{j_{3}}(s) d s\right)^{2} d s_{2} d s_{3} \zeta_{j_{1}}^{\left(i_{1}\right)} \zeta_{j_{2}}^{\left(i_{2}\right)} \\
& =\underset{p \rightarrow \infty}{\operatorname{li} . m} \sum_{j_{1}, j_{2}=0}^{p} \frac{1}{2} \int_{t}^{T} \phi_{j_{1}}\left(s_{3}\right) \int_{s_{3}}^{T} \phi_{j_{2}}\left(s_{2}\right)\left(T-s_{2}\right) d s_{2} d s_{3} \zeta_{j_{1}}^{\left(i_{1}\right)} \zeta_{j_{2}}^{\left(i_{2}\right)}-\Delta_{6}^{\left(i_{1} i_{2}\right)} \\
& =\underset{p \rightarrow \infty}{\lim } \sum_{j_{1}, j_{2}=0}^{p} \frac{1}{2} \int_{t}^{T} \phi_{j_{2}}\left(s_{2}\right)\left(T-s_{2}\right) \int_{t}^{s_{2}} \phi_{j_{1}}\left(s_{3}\right) d s_{3} d s_{2} \zeta_{j_{1}}^{\left(i_{1}\right)} \zeta_{j_{2}}^{\left(i_{2}\right)}-\Delta_{6}^{\left(i_{1} i_{2}\right)} \\
& =\frac{1}{2} \int_{t}^{T}\left(T-s_{2}\right) \int_{t}^{s_{2}} d \mathbf{w}_{s_{3}}^{\left(i_{1}\right)} d \mathbf{w}_{s_{2}}^{\left(i_{2}\right)} \\
& +\frac{1}{2} \mathbf{1}_{\left\{i_{1}=i_{2} \neq 0\right\}} \sum_{j_{2}=0}^{\infty} \int_{t}^{T} \phi_{j_{2}}\left(s_{2}\right)\left(T-s_{2}\right) \int_{t}^{s_{2}} \phi_{j_{2}}\left(s_{3}\right) d s_{3} d s_{2}-\Delta_{6}^{\left(i_{1} i_{2}\right)} \\
& =\frac{1}{2} \int_{t}^{T} \int_{t}^{s_{1}} \int_{t}^{s_{2}} d \mathbf{w}_{s}^{\left(i_{1}\right)} d \mathbf{w}_{s_{2}}^{\left(i_{2}\right)} d s_{1}+\frac{1}{4} \mathbf{1}_{\left\{i_{1}=i_{2} \neq 0\right\}} \int_{t}^{T}\left(T-s_{2}\right) d s_{2}-\Delta_{6}^{\left(i_{1} i_{2}\right)}
\end{aligned}
$$


with probability 1 .

We proceed to $B_{1}, B_{2}, B_{3}$. We have:

$$
\begin{aligned}
B_{1} & =\underset{p \rightarrow \infty}{\lim .} \sum_{j_{1}, j_{4}=0}^{p} \frac{1}{2} \int_{t}^{T} \phi_{j_{4}}(s) \int_{t}^{s} \phi_{j_{4}}\left(s_{1}\right)\left(\int_{t}^{s_{1}} \phi_{j_{1}}\left(s_{2}\right) d s_{2}\right)^{2} d s_{1} d s \\
& =\operatorname{li}_{p \rightarrow \infty} . \sum_{j_{4}=0}^{p} \frac{1}{2} \int_{t}^{T} \phi_{j_{4}}(s) \int_{t}^{s} \phi_{j_{4}}\left(s_{1}\right)\left(s_{1}-t\right) d s_{1} d s-\lim _{p \rightarrow \infty} \sum_{j_{4}=0}^{p} a_{j_{4} j_{4}}^{p} \\
& =\frac{1}{4} \int_{t}^{T}\left(s_{1}-t\right) d s_{1}-\lim _{p \rightarrow \infty} \sum_{j_{4}=0}^{p} a_{j_{4} j_{4}}^{p} .
\end{aligned}
$$

The next formula is

$$
\begin{aligned}
B_{2}= & l_{p \rightarrow \infty} . m_{j_{4}, j_{3}=0}^{p}\left(\frac{1}{2} \int_{t}^{T} \phi_{j_{3}}\left(s_{3}\right)\left(\int_{t}^{s_{3}} \phi_{j_{4}}(s) d s\right)^{2} \int_{t}^{s_{3}} \phi_{j_{3}}\left(s_{1}\right) d s_{1} d s_{3}\right. \\
& -\frac{1}{2} \int_{t}^{T} \phi_{j_{3}}\left(s_{1}\right) \int_{t}^{s_{1}} \phi_{j_{3}}\left(s_{2}\right)\left(\int_{t}^{s_{2}} \phi_{j_{4}}\left(s_{3}\right) d s_{3}\right)^{2} d s_{2} d s_{1} \\
& \left.-\frac{1}{2} \int_{t}^{T} \phi_{j_{3}}\left(s_{1}\right) \int_{t}^{s_{1}} \phi_{j_{3}}(s)\left(\int_{s}^{s_{1}} \phi_{j_{4}}\left(s_{2}\right) d s_{2}\right)^{2} d s d s_{1}\right) \\
= & \sum_{j_{3}=0}^{p} \frac{1}{2} \int_{t}^{T} \phi_{j_{3}}\left(s_{3}\right)\left(s_{3}-t\right) \int_{t}^{s_{3}} \phi_{j_{3}}\left(s_{1}\right) d s_{1} d s_{3}-\lim _{p \rightarrow \infty} \sum_{j_{3}=0}^{p} b_{j_{3} j_{3}}^{p} \\
& -\sum_{j_{3}=0}^{p} \frac{1}{2} \int_{t}^{T} \phi_{j_{3}}\left(s_{1}\right) \int_{t}^{s_{1}}\left(s_{2}-t\right) \phi_{j_{3}}\left(s_{2}\right) d s_{2} d s_{1}+\lim _{p \rightarrow \infty} \sum_{j_{3}=0}^{p} a_{j_{3} j_{3}}^{p} \\
& -\sum_{j_{3}=0}^{p} \frac{1}{2} \int_{t}^{T} \phi_{j_{3}}\left(s_{1}\right) \int_{t}^{s_{1}} \phi_{j_{3}}(s)\left(s_{1}-t+t-s\right) d s d s_{1}+\lim _{p \rightarrow \infty} \sum_{j_{3}=0}^{p} c_{j_{3} j_{3}}^{p} \\
= & \lim _{p \rightarrow \infty} \sum_{j_{3}=0}^{p} a_{j_{3} j_{3}}^{p}+\lim _{p \rightarrow \infty} \sum_{j_{3}=0}^{p} c_{j_{3} j_{3}}^{p}-\lim _{p \rightarrow \infty} \sum_{j_{3}=0}^{p} b_{j_{3} j_{3}}^{p} .
\end{aligned}
$$

Moreover,

$$
\begin{aligned}
B_{2}+B_{3} & =\underset{p \rightarrow \infty}{\lim } \sum_{j_{4}, j_{3}=0}^{p}\left(C_{j_{3} j_{4} j_{3} j_{4}}+C_{j_{3} j_{4} j_{4} j_{3}}\right) \\
& =\lim _{p \rightarrow \infty} \sum_{j_{4}, j_{3}=0}^{p} \int_{t}^{T} \phi_{j_{3}}(s) \int_{t}^{s} \phi_{j_{4}}\left(s_{1}\right) \int_{t}^{s_{1}} \phi_{j_{4}}\left(s_{2}\right) \int_{t}^{s_{1}} \phi_{j_{3}}\left(s_{3}\right) d s_{3} d s_{2} d s_{1} d s \\
& =\lim _{p \rightarrow \infty} \sum_{j_{4}, j_{3}=0}^{p} \int_{t}^{T} \phi_{j_{4}}\left(s_{1}\right) \int_{t}^{s_{1}} \phi_{j_{4}}\left(s_{2}\right) \int_{t}^{s_{1}} \phi_{j_{3}}\left(s_{3}\right) d s_{3} d s_{2} \int_{s_{1}}^{T} \phi_{j_{3}}(s) d s d s_{1}
\end{aligned}
$$




$$
\begin{aligned}
= & \underset{p \rightarrow \infty}{\text { i.m. }}{ }_{j_{4}, j_{3}=0}^{p}\left(\int_{t}^{T} \phi_{j_{4}}\left(s_{1}\right) \int_{t}^{s_{1}} \phi_{j_{4}}\left(s_{3}\right) \int_{t}^{T} \phi_{j_{3}}\left(s_{2}\right) d s_{2} \int_{s_{1}}^{T} \phi_{j_{3}}(s) d s d s_{3} d s_{1}\right. \\
& \left.\quad-\int_{t}^{T} \phi_{j_{4}}\left(s_{1}\right) \int_{t}^{s_{1}} \phi_{j_{4}}\left(s_{3}\right)\left(\int_{s_{1}}^{T} \phi_{j_{3}}(s) d s\right)^{2} d s_{3} d s_{1}\right) \\
= & \sum_{j_{4}=0}^{\infty} \int_{t}^{T} \phi_{j_{4}}\left(s_{1}\right)\left(T-s_{1}\right) \int_{t}^{s_{1}} \phi_{j_{4}}\left(s_{3}\right) d s_{3} d s_{1} \\
& -\sum_{j_{4}=0}^{\infty} \int_{t}^{T} \phi_{j_{4}}\left(s_{1}\right)\left(T-s_{1}\right) \int_{t}^{s_{1}} \phi_{j_{4}}\left(s_{3}\right) d s_{3} d s_{1}+2 \lim _{p \rightarrow \infty} \sum_{j_{4}=0}^{p} f_{j_{4} j_{4}}^{p}=2 \lim _{p \rightarrow \infty} \sum_{j_{4}=0}^{p} f_{j_{4} j_{4}}^{p} .
\end{aligned}
$$

This is why

$$
B_{3}=2 \lim _{p \rightarrow \infty} \sum_{j_{3}=0}^{p} f_{j_{3} j_{3}}^{p}-\lim _{p \rightarrow \infty} \sum_{j_{3}=0}^{p} a_{j_{3} j_{3}}^{p}-\lim _{p \rightarrow \infty} \sum_{j_{3}=0}^{p} c_{j_{3} j_{3}}^{p}+\lim _{p \rightarrow \infty} \sum_{j_{3}=0}^{p} b_{j_{3} j_{3}}^{p} .
$$

Substituting relations 62 -71) into 61), we obtain

$$
\begin{aligned}
& \underset{p \rightarrow \infty}{\lim .}{ }_{j_{1}, j_{2}, j_{3}, j_{4}=0}^{p} C_{j_{4} j_{3} j_{2} j_{1}} \zeta_{j_{1}}^{\left(i_{1}\right)} \zeta_{j_{2}}^{\left(i_{2}\right)} \zeta_{j_{3}}^{\left(i_{3}\right)} \zeta_{j_{4}}^{\left(i_{4}\right)}=J\left[\psi^{(4)}\right]_{T, t} \\
& \quad+\frac{1}{2} \mathbf{1}_{\left\{i_{1}=i_{2} \neq 0\right\}} \int_{t}^{T} \int_{t}^{s} \int_{t}^{s} d s_{2} d \mathbf{w}_{s_{1}}^{\left(i_{3}\right)} d \mathbf{w}_{s}^{\left(i_{4}\right)} \\
& \quad+\frac{1}{2} \mathbf{1}_{\left\{i_{2}=i_{3} \neq 0\right\}} \int_{t}^{T} \int_{t}^{s_{2}} \int_{t}^{s_{1}} d \mathbf{w}_{s}^{\left(i_{1}\right)} d s_{1} d \mathbf{w}_{s_{2}}^{\left(i_{4}\right)}+\frac{1}{2} \mathbf{1}_{\left\{i_{3}=i_{4} \neq 0\right\}} \int_{t}^{T} \int_{t}^{s_{1}} \int_{t}^{s_{2}} d \mathbf{w}_{s}^{\left(i_{1}\right)} d \mathbf{w}_{s_{2}}^{\left(i_{2}\right)} d s_{1} \\
& \quad+\frac{1}{4} \mathbf{1}_{\left\{i_{1}=i_{2} \neq 0\right\}} \mathbf{1}_{\left\{i_{3}=i_{4} \neq 0\right\}} \int_{t}^{T} \int_{t}^{s_{1}} d s_{2} d s_{1}+R=J^{*}\left[\psi^{(4)}\right]_{T, t}+R
\end{aligned}
$$

with probability 1 , where

$$
\begin{aligned}
R= & -\mathbf{1}_{\left\{i_{1}=i_{2} \neq 0\right\}} \Delta_{1}^{\left(i_{3} i_{4}\right)}+\mathbf{1}_{\left\{i_{1}=i_{3} \neq 0\right\}}\left(-\Delta_{2}^{\left(i_{2} i_{4}\right)}+\Delta_{1}^{\left(i_{2} i_{4}\right)}+\Delta_{3}^{\left(i_{2} i_{4}\right)}\right) \\
& +\mathbf{1}_{\left\{i_{1}=i_{4} \neq 0\right\}}\left(\Delta_{4}^{\left(i_{2} i_{3}\right)}-\Delta_{5}^{\left(i_{2} i_{3}\right)}+\Delta_{6}^{\left(i_{2} i_{3}\right)}\right)-\mathbf{1}_{\left\{i_{2}=i_{3} \neq 0\right\}} \Delta_{3}^{\left(i_{1} i_{4}\right)} \\
& +\mathbf{1}_{\left\{i_{2}=i_{4} \neq 0\right\}}\left(-\Delta_{4}^{\left(i_{1} i_{3}\right)}+\Delta_{5}^{\left(i_{1} i_{3}\right)}+\Delta_{6}^{\left(i_{1} i_{3}\right)}\right)-\mathbf{1}_{\left\{i_{3}=i_{4} \neq 0\right\}} \Delta_{6}^{\left(i_{1} i_{2}\right)} \\
& +\mathbf{1}_{\left\{i_{1}=i_{2} \neq 0\right\}} \mathbf{1}_{\left\{i_{3}=i_{4} \neq 0\right\}} \lim _{p \rightarrow \infty} \sum_{j_{3}=0}^{p} a_{j_{3} j_{3}}^{p} \\
& -\mathbf{1}_{\left\{i_{1}=i_{3} \neq 0\right\}} \mathbf{1}_{\left\{i_{2}=i_{4} \neq 0\right\}}\left(\lim _{p \rightarrow \infty} \sum_{j_{3}=0}^{p} a_{j_{3} j_{3}}^{p}+\lim _{p \rightarrow \infty} \sum_{j_{3}=0}^{p} c_{j_{3} j_{3}}^{p}-\lim _{p \rightarrow \infty} \sum_{j_{3}=0}^{p} b_{j_{3} j_{3}}^{p}\right) \\
& -\mathbf{1}_{\left\{i_{1}=i_{4} \neq 0\right\}} \mathbf{1}_{\left\{i_{2}=i_{3} \neq 0\right\}} \\
& \cdot\left(2 \lim _{p \rightarrow \infty} \sum_{j_{3}=0}^{p} f_{j_{3} j_{3}}^{p}-\lim _{p \rightarrow \infty} \sum_{j_{3}=0}^{p} a_{j_{3} j_{3}}^{p}-\lim _{p \rightarrow \infty} \sum_{j_{3}=0}^{p} c_{j_{3} j_{3}}^{p}+\lim _{p \rightarrow \infty} \sum_{j_{3}=0}^{p} b_{j_{3} j_{3}}^{p}\right) .
\end{aligned}
$$


It follows from $(72)$ and $(73)$ that we shall complete the proof of Theorem 4 if

$$
\begin{aligned}
& \Delta_{k}^{(i j)}=0 \quad \text { with probability } 1 \text { and } \\
& \sum_{j_{3}=0}^{p} a_{j_{3} j_{3}}^{p} \rightarrow 0, \quad \sum_{j_{3}=0}^{p} b_{j_{3} j_{3}}^{p} \rightarrow 0, \quad \sum_{j_{3}=0}^{p} c_{j_{3} j_{3}}^{p} \rightarrow 0, \quad \sum_{j_{3}=0}^{p} f_{j_{3} j_{3}}^{p} \rightarrow 0 \quad \text { as } \quad p \rightarrow \infty,
\end{aligned}
$$

where $k=1,2, \ldots, 6, i, j=0,1, \ldots, m$.

We consider the case of Legendre polynomials. We are going to prove that $\Delta_{1}^{\left(i_{3} i_{4}\right)}=0$ with probability 1. We have:

$$
\begin{aligned}
& \mathrm{M}\left(\left(\sum_{j_{3}, j_{4}=0}^{p} a_{j_{4} j_{3}}^{p} \zeta_{j_{3}}^{\left(i_{3}\right)} \zeta_{j_{4}}^{\left(i_{4}\right)}\right)^{2}\right) \\
& \quad=\sum_{j_{3}^{\prime}=0}^{p} \sum_{j_{3}=0}^{j_{3}^{\prime}-1}\left(2 a_{j_{3} j_{3}}^{p} a_{j_{3}^{\prime} j_{3}^{\prime}}^{p}+\left(a_{j_{3} j_{3}^{\prime}}^{p}\right)^{2}+2 a_{j_{3} j_{3}^{\prime}}^{p} a_{j_{3}^{\prime} j_{3}}^{p}+\left(a_{j_{3}^{\prime} j_{3}}^{p}\right)^{2}\right)+3 \sum_{j_{3}^{\prime}=0}^{p}\left(a_{j_{3}^{\prime} j_{3}^{\prime}}^{p}\right)^{2} \\
& \quad=\left(\sum_{j_{3}=0}^{p} a_{j_{3} j_{3}}^{p}\right)^{2}+\sum_{j_{3}^{\prime}=0}^{p} \sum_{j_{3}=0}^{j_{3}^{\prime}-1}\left(a_{j_{3} j_{3}^{\prime}}^{p}+a_{j_{3}^{\prime} j_{3}}^{p}\right)^{2}+2 \sum_{j_{3}^{\prime}=0}^{p}\left(a_{j_{3}^{\prime} j_{3}^{\prime}}^{p}\right)^{2}, \quad i_{3}=i_{4} \neq 0, \\
& \mathrm{M}\left(\left(\sum_{j_{3}, j_{4}=0}^{p} a_{j_{4} j_{3}}^{p} \zeta_{j_{3}}^{\left(i_{3}\right)} \zeta_{j_{4}}^{\left(i_{4}\right)}\right)^{2}\right)=\sum_{j_{3}, j_{4}=0}^{p}\left(a_{j_{4} j_{3}}^{p}\right)^{2}, \quad i_{3} \neq i_{4}, i_{3} \neq 0, i_{4} \neq 0, \\
& (T-t) \sum_{j_{4}=0}^{p}\left(a_{j_{4}, 0}^{p}\right)^{2} \text { as } i_{3}=0, i_{4} \neq 0 \\
& (T-t) \sum_{j_{3}=0}^{p}\left(a_{0, j_{3}}^{p}\right)^{2} \text { as } i_{4}=0, i_{3} \neq 0 \\
& \left.\left.(T-t)^{2}\left(a_{00}^{p}\right)^{2} \text { as } i_{j_{4} j_{3}} \zeta_{j_{3}}^{\left(i_{3}\right)} \zeta_{j_{4}}^{\left(i_{4}\right)}\right)^{2}\right)=i_{4}=0 .
\end{aligned}
$$

We consider the case $i_{3}=i_{4} \neq 0$ :

$$
\begin{aligned}
a_{j_{4} j_{3}}^{p}= & \frac{(T-t)^{2} \sqrt{\left(2 j_{4}+1\right)\left(2 j_{3}+1\right)}}{32} \int_{-1}^{1} P_{j_{4}}(y) \int_{-1}^{y} P_{j_{3}}\left(y_{1}\right) \sum_{j_{1}=p+1}^{\infty}\left(2 j_{1}+1\right)\left(\int_{-1}^{y_{1}} P_{j_{1}}\left(y_{2}\right) d y_{2}\right)^{2} d y_{1} d y \\
= & \frac{(T-t)^{2} \sqrt{\left(2 j_{4}+1\right)\left(2 j_{3}+1\right)}}{32} \\
& \cdot \int_{-1}^{1} P_{j_{3}}\left(y_{1}\right) \sum_{j_{1}=p+1}^{\infty} \frac{1}{2 j_{1}+1}\left(P_{j_{1}+1}\left(y_{1}\right)-P_{j_{1}-1}\left(y_{1}\right)\right)^{2} \int_{y_{1}}^{1} P_{j_{4}}(y) d y d y_{1} \\
= & \frac{(T-t)^{2} \sqrt{2 j_{3}+1}}{32 \sqrt{2 j_{4}+1}} \int_{-1}^{1} P_{j_{3}}\left(y_{1}\right)\left(P_{j_{4}-1}\left(y_{1}\right)-P_{j_{4}+1}\left(y_{1}\right)\right) \\
& \cdot \sum_{j_{1}=p+1}^{\infty} \frac{1}{2 j_{1}+1}\left(P_{j_{1}+1}\left(y_{1}\right)-P_{j_{1}-1}\left(y_{1}\right)\right)^{2} d y_{1}, \quad j_{4} \neq 0, \\
a_{j_{4} j_{3}}^{p}= & \frac{(T-t)^{2} \sqrt{2 j_{3}+1}}{32} \cdot \int_{-1}^{1} P_{j_{3}}\left(y_{1}\right)\left(1-y_{1}\right) \sum_{j_{1}=p+1}^{\infty} \frac{1}{2 j_{1}+1}\left(P_{j_{1}+1}\left(y_{1}\right)-P_{j_{1}-1}\left(y_{1}\right)\right)^{2} d y_{1}, \quad j_{4}=0 .
\end{aligned}
$$


By (28) and the estimate $\left|P_{j_{4}-1}(y)-P_{j_{4}+1}(y)\right| \leqslant 2, y \in[-1,1]$ we obtain

$$
\begin{aligned}
& \left|a_{j_{4} j_{3}}^{p}\right| \leqslant \frac{C_{0}}{\sqrt{j_{4}}} \sum_{j_{1}=p+1}^{\infty} \frac{1}{j_{1}^{2}} I_{\frac{3}{4}}(1) \leqslant \frac{C_{1}}{p \sqrt{j_{4}}}, \quad j_{4} \neq 0, \\
& \left|a_{0, j_{3}}^{p}\right| \leqslant C \sum_{j_{1}=p+1}^{\infty} \frac{1}{j_{1}^{2}} I_{\frac{3}{4}}(1) \leqslant \frac{C_{1}}{p}, \quad\left|a_{00}^{p}\right| \leqslant C \sum_{j_{1}=p+1}^{\infty} \frac{1}{j_{1}^{2}} I_{\frac{1}{2}}(1) \leqslant \frac{C_{1}}{p} .
\end{aligned}
$$

Taking 75)-79 into consideration, we write

$$
\begin{aligned}
\mathrm{M}\left(\left(\sum_{j_{3}, j_{4}=0}^{p} a_{j_{4} j_{3}}^{p} \zeta_{j_{3}}^{\left(i_{3}\right)} \zeta_{j_{4}}^{\left(i_{4}\right)}\right)^{2}\right)= & \left(a_{00}^{p}+\sum_{j_{3}=1}^{p} a_{j_{3} j_{3}}^{p}\right)^{2}+\sum_{j_{3}^{\prime}=1}^{p}\left(a_{0, j_{3}^{\prime}}^{p}+a_{j_{3}^{\prime}, 0}^{p}\right)^{2} \\
& +\sum_{j_{3}^{\prime}=1}^{p} \sum_{j_{3}=1}^{j_{3}^{\prime}-1}\left(a_{j_{3} j_{3}^{\prime}}^{p}+a_{j_{3}^{\prime} j_{3}}^{p}\right)^{2}+2\left(\sum_{j_{3}^{\prime}=1}^{p}\left(a_{j_{3}^{\prime} j_{3}^{\prime}}^{p}\right)^{2}+\left(a_{00}\right)^{2}\right) \\
\leqslant & K_{0}\left(\frac{1}{p}+\frac{1}{p} \sum_{j_{3}=1}^{p} \frac{1}{\sqrt{j_{3}}}\right)^{2}+\frac{K_{1}}{p}+K_{2} \sum_{j_{3}^{\prime}=1}^{p} \sum_{j_{3}=1}^{j_{3}^{\prime}-1} \frac{1}{p^{2}}\left(\frac{1}{\sqrt{j_{3}^{\prime}}}+\frac{1}{\sqrt{j_{3}}}\right)^{2} \\
\leqslant & K_{0}\left(\frac{1}{p}+\frac{1}{p} \int_{0}^{p} \frac{d x}{\sqrt{x}}\right)^{2}+\frac{K_{1}}{p}+\frac{K_{3}}{p} \sum_{j_{3}=1}^{p} \frac{1}{j_{3}} \\
\leqslant & K_{0}\left(\frac{1}{p}+\frac{2}{\sqrt{p}}\right)^{2}+\frac{K_{1}}{p}+\frac{K_{3}}{p}\left(1+\int_{1}^{p} \frac{d x}{x}\right)^{2} \\
\leqslant & \frac{K_{4}}{p}+\frac{K_{3}(\ln p+1)}{p} \rightarrow 0 \quad \text { as } p \rightarrow \infty, \quad i_{3}=i_{4} \neq 0 .
\end{aligned}
$$

A similar result for the cases (76), (77) is implied by estimates $(78),(79)$. This is why

$$
\Delta_{1}^{\left(i_{3} i_{4}\right)}=0 \quad \text { with probability } 1 .
$$

It is easy to see that the formulae

$$
\Delta_{2}^{\left(i_{2} i_{4}\right)}=0, \quad \Delta_{4}^{\left(i_{1} i_{3}\right)}=0, \quad \Delta_{6}^{\left(i_{1} i_{3}\right)}=0 \quad \text { with probability } 1
$$

can be obtained similar to the proof of relation (80). Moreover, by estimates (78), (79) we obtain

$$
\lim _{p \rightarrow \infty} \sum_{j_{3}=0}^{p} a_{j_{3} j_{3}}^{p}=0 .
$$

Similar to the proof of 82 , we find:

$$
\lim _{p \rightarrow \infty} \sum_{j_{3}=0}^{p} b_{j_{3} j_{3}}^{p}=0, \quad \lim _{p \rightarrow \infty} \sum_{j_{3}=0}^{p} f_{j_{3} j_{3}}^{p}=0 .
$$

We consider $\Delta_{3}^{\left(i_{2} i_{4}\right)}$ :

$$
\Delta_{3}^{\left(i_{2} i_{4}\right)}=\Delta_{4}^{\left(i_{2} i_{4}\right)}+\Delta_{6}^{\left(i_{2} i_{4}\right)}-\Delta_{7}^{\left(i_{2} i_{4}\right)}=-\Delta_{7}^{\left(i_{2} i_{4}\right)}
$$


with probability 1 , where

$$
\begin{aligned}
\Delta_{7}^{\left(i_{2} i_{4}\right)} & =\lim _{p \rightarrow \infty} \sum_{j_{2}, j_{4}=0}^{p} g_{j_{4} j_{2}}^{p} \zeta_{j_{2}}^{\left(i_{2}\right)} \zeta_{j_{4}}^{\left(i_{4}\right)}, \\
g_{j_{4} j_{2}}^{p} & =\int_{t}^{T} \phi_{j_{4}}(s) \int_{t}^{s} \phi_{j_{2}}\left(s_{1}\right) \sum_{j_{1}=p+1}^{\infty}\left(\int_{s_{1}}^{T} \phi_{j_{1}}\left(s_{2}\right) d s_{2} \int_{s}^{T} \phi_{j_{1}}\left(s_{2}\right) d s_{2}\right) d s_{1} d s \\
& =\sum_{j_{1}=p+1}^{\infty} \int_{t}^{T} \phi_{j_{4}}(s) \int_{s}^{T} \phi_{j_{1}}\left(s_{2}\right) d s_{2} \int_{t}^{T} \phi_{j_{2}}\left(s_{1}\right) \int_{s_{1}}^{T} \phi_{j_{1}}\left(s_{2}\right) d s_{2} d s_{1} d s .
\end{aligned}
$$

Identity 85 is implied by the estimate:

$$
\left|g_{j_{4} j_{2}}^{p}\right| \leqslant K \sum_{j_{1}=p+1}^{\infty} \frac{1}{j_{1}^{2}} \int_{-1}^{1} \frac{I_{\frac{1}{2}}(y)}{\left(1-y^{2}\right)^{\frac{1}{2}}} d y \leqslant \frac{K_{1}}{p} .
$$

We observe that

$$
\begin{aligned}
& g_{j_{4} j_{4}}^{p}=\sum_{j_{1}=p+1}^{\infty} \frac{1}{2}\left(\int_{t}^{T} \phi_{j_{4}}(s) \int_{s}^{T} \phi_{j_{1}}\left(s_{2}\right) d s_{2} d s\right)^{2} \\
& g_{j_{4} j_{2}}^{p}+g_{j_{2} j_{4}}^{p}=\sum_{j_{1}=p+1}^{\infty} \int_{t}^{T} \phi_{j_{4}}(s) \int_{s}^{T} \phi_{j_{1}}\left(s_{2}\right) d s_{2} d s \int_{t}^{T} \phi_{j_{2}}(s) \int_{s}^{T} \phi_{j_{1}}\left(s_{2}\right) d s_{2} d s
\end{aligned}
$$

and moreover, as $j_{4}, j_{2} \leqslant p$,

$$
\begin{aligned}
g_{j_{4} j_{2}}^{p}= & \frac{(T-t)^{2} \sqrt{\left(2 j_{4}+1\right)\left(2 j_{2}+1\right)}}{16} \sum_{j_{1}=p+1}^{\infty} \frac{1}{2 j_{1}+1} \\
& \cdot \int_{-1}^{1} P_{j_{4}}\left(y_{1}\right)\left(P_{j_{1}-1}\left(y_{1}\right)-P_{j_{1}+1}\left(y_{1}\right)\right) \int_{-1}^{y_{1}} P_{j_{2}}(y)\left(P_{j_{1}-1}(y)-P_{j_{1}+1}(y)\right) d y d y_{1} .
\end{aligned}
$$

By the orthonormality of the Legendre polynomials we obtain

$$
\begin{aligned}
& g_{j_{4} j_{2}}^{p}+g_{j_{2} j_{4}}^{p}=\frac{(T-t)^{2} \sqrt{\left(2 j_{4}+1\right)\left(2 j_{2}+1\right)}}{16} \sum_{j_{1}=p+1}^{\infty} \frac{1}{2 j_{1}+1} \\
& \cdot \int_{-1}^{1} P_{j_{4}}\left(y_{1}\right)\left(P_{j_{1}-1}\left(y_{1}\right)-P_{j_{1}+1}\left(y_{1}\right)\right) d y_{1} \int_{-1}^{1} P_{j_{2}}(y)\left(P_{j_{1}-1}(y)-P_{j_{1}+1}(y)\right) d y \\
& =\frac{(T-t)^{2}(2 p+1)}{16} \frac{1}{2 p+3}\left(\int_{-1}^{1} P_{p}^{2}\left(y_{1}\right) d y_{1}\right)^{2} \cdot \begin{cases}1 & \text { as } j_{2}=j_{4}=p \\
0 & \text { otherwise }\end{cases} \\
& =\frac{(T-t)^{2}}{4(2 p+3)(2 p+1)} \cdot \begin{cases}1 & \text { as } j_{2}=j_{4}=p \\
0 & \text { otherwise }\end{cases} \\
& g_{j_{4} j_{4}}^{p}=\left.\frac{1}{2}\left(g_{j_{4} j_{2}}^{p}+g_{j_{2} j_{4}}^{p}\right)\right|_{j_{2}=j_{4}}=\frac{(T-t)^{2}}{8(2 p+3)(2 p+1)} \cdot \begin{cases}1 & \text { as } j_{4}=p \\
0 & \text { otherwise }\end{cases}
\end{aligned}
$$


By (75), (88) and (89) we obtain

$$
\begin{aligned}
\mathrm{M}\left(\left(\sum_{j_{2}, j_{4}=0}^{p} g_{j_{4} j_{2}}^{p} \zeta_{j_{2}}^{\left(i_{2}\right)} \zeta_{j_{4}}^{\left(i_{4}\right)}\right)^{2}\right) & =\left(\sum_{j_{3}=0}^{p} g_{j_{3} j_{3}}^{p}\right)^{2}+\sum_{j_{3}^{\prime}=0}^{p} \sum_{j_{3}=0}^{j_{3}^{\prime}-1}\left(g_{j_{3} j_{3}^{\prime}}^{p}+g_{j_{3}^{\prime} j_{3}}^{p}\right)^{2}+2 \sum_{j_{3}^{\prime}=0}^{p}\left(g_{j_{3}^{\prime} j_{3}^{\prime}}^{p}\right)^{2} \\
& \leqslant\left(\frac{(T-t)^{2}}{8(2 p+3)(2 p+1)}\right)^{2}+0+2\left(\frac{(T-t)^{2}}{8(2 p+3)(2 p+1)}\right)^{2} \rightarrow 0
\end{aligned}
$$

as $p \rightarrow \infty, i_{2}=i_{4} \neq 0$.

We proceed to the case $i_{2} \neq i_{4}, i_{2} \neq 0, i_{4} \neq 0$. It is easy to see that

$$
g_{j_{4} j_{2}}^{p}=\int_{t}^{T} \phi_{j_{4}}(s) \int_{t}^{s} \phi_{j_{2}}\left(s_{1}\right) F^{p}\left(s, s_{1}\right) d s_{1} d s=\int_{[t, T]^{2}} K_{p}\left(s, s_{1}\right) \phi_{j_{4}}(s) \phi_{j_{2}}\left(s_{1}\right) d s_{1} d s
$$

is the Fourier coefficients in the double Fourier-Legendre series of the function

$$
K_{p}\left(s, s_{1}\right)=\mathbf{1}_{\left\{s_{1}<s\right\}} F^{p}\left(s, s_{1}\right),
$$

where

$$
F^{p, n}\left(s, s_{1}\right) \stackrel{\text { def }}{=} \sum_{j_{1}=p+1}^{n} \int_{s_{1}}^{T} \phi_{j_{1}}\left(s_{2}\right) d s_{2} \int_{s}^{T} \phi_{j_{1}}\left(s_{2}\right) d s_{2}, \quad F^{p, \infty}\left(s, s_{1}\right) \stackrel{\text { def }}{=} F^{p}\left(s, s_{1}\right) .
$$

In this case, the Parseval identity reads as

$$
\lim _{p_{1} \rightarrow \infty} \sum_{j_{4}, j_{2}=0}^{p_{1}}\left(g_{j_{4} j_{2}}^{p}\right)^{2}=\int_{[t, T]^{2}}\left(K_{p}\left(s, s_{1}\right)\right)^{2} d s_{1} d s=\int_{t}^{T} \int_{t}^{s}\left(F^{p}\left(s, s_{1}\right)\right)^{2} d s_{1} d s .
$$

By (28) we obtain:

$$
\begin{aligned}
\left|\int_{s_{1}}^{T} \phi_{j_{1}}(\theta) d \theta\right| & =\frac{1}{2} \sqrt{2 j_{1}+1} \sqrt{T-t}\left|\int_{z\left(s_{1}\right)}^{1} P_{j_{1}}(y) d y\right| \\
& =\frac{\sqrt{T-t}}{2 \sqrt{2 j_{1}+1}}\left|P_{j_{1}-1}\left(z\left(s_{1}\right)\right)-P_{j_{1}+1}\left(z\left(s_{1}\right)\right)\right| \leqslant \frac{K}{j_{1}} f_{\frac{1}{4}}\left(s_{1}\right), \quad s_{1} \in(t, T) .
\end{aligned}
$$

Employing (30) and (92), we find:

$$
\left(F^{p}\left(s, s_{1}\right)\right)^{2} \leqslant \frac{C}{p^{2}} f_{\frac{1}{2}}(s) f_{\frac{1}{2}}\left(s_{1}\right), \quad s, s_{1} \in(t, T) .
$$

It follows from (93) that $\left|F^{p}\left(s, s_{1}\right)\right| \leqslant K / p$ in the domain

$$
D_{\varepsilon}=\left\{\left(s, s_{1}\right): s \in[t+\varepsilon, T-\varepsilon], s_{1} \in[t+\varepsilon, s]\right\},
$$

where $\varepsilon>0$ is a sufficiently small fixed number. Then the uniform convergence holds:

$$
F^{-1, p}\left(s, s_{1}\right) \rightarrow F^{-1}\left(s, s_{1}\right)
$$

on the domain $D_{\varepsilon}$ as $p \rightarrow \infty$. By the continuity of the left hand side in (94) we get the continuity of the limiting function in the right hand side in (94) on the set $D_{\varepsilon}$. Employing this fact and 
(93), we obtain:

$$
\begin{aligned}
& \int_{t}^{T} \int_{t}^{s}\left(F^{p}\left(s, s_{1}\right)\right)^{2} d s_{1} d s=\lim _{\varepsilon \rightarrow+0} \int_{t+\varepsilon}^{T-\varepsilon} \int_{t+\varepsilon}^{s}\left(F^{p}\left(s, s_{1}\right)\right)^{2} d s_{1} d s \\
& \quad \leqslant \frac{C}{p^{2}} \lim _{\varepsilon \rightarrow+0} \int_{t+\varepsilon}^{T-\varepsilon} f_{\frac{1}{2}}(s) \int_{t+\varepsilon}^{s} f_{\frac{1}{2}}\left(s_{1}\right) d s_{1} d s=\frac{C}{p^{2}} \int_{t}^{T} f_{\frac{1}{2}}(s) \int_{t}^{s} f_{\frac{1}{2}}\left(s_{1}\right) d s_{1} d s \\
& \quad=\frac{C(T-t)^{2}}{4 p^{2}} \int_{-1}^{1} \frac{I_{\frac{1}{2}}(y)}{\left(1-y^{2}\right)^{\frac{1}{2}}} d y<\frac{K_{1}}{p^{2}} .
\end{aligned}
$$

By (95) and (91) we get:

$$
0 \leqslant \sum_{j_{2}, j_{4}=0}^{p}\left(g_{j_{4} j_{2}}^{p}\right)^{2} \leqslant \lim _{p_{1} \rightarrow \infty} \sum_{j_{2}, j_{4}=0}^{p_{1}}\left(g_{j_{4} j_{2}}^{p}\right)^{2}=\sum_{j_{2}, j_{4}=0}^{\infty}\left(g_{j_{4} j_{2}}^{p}\right)^{2} \leqslant \frac{K_{1}}{p^{2}} \rightarrow 0
$$

as $p \rightarrow \infty$. This completes the case $i_{2} \neq i_{4}, i_{2} \neq 0, i_{4} \neq 0$.

It is easy to obtain a similar result for the cases $i_{2}=0, i_{4} \neq 0 ; i_{4}=0, i_{2} \neq 0$; and $i_{2}=0$, $i_{4}=0$. Then $\Delta_{7}^{\left(i_{2} i_{4}\right)}=0$ and $\Delta_{3}^{\left(i_{2} i_{4}\right)}=0$ with probability 1 .

We consider $\Delta_{5}^{\left(i_{1} i_{3}\right)}$ :

$$
\Delta_{5}^{\left(i_{1} i_{3}\right)}=\Delta_{4}^{\left(i_{1} i_{3}\right)}+\Delta_{6}^{\left(i_{1} i_{3}\right)}-\Delta_{8}^{\left(i_{1} i_{3}\right)} \text { with probability } 1,
$$

where

$$
\Delta_{8}^{\left(i_{1} i_{3}\right)}=\underset{p \rightarrow \infty}{\operatorname{li} . \mathrm{m}} \sum_{j_{3}, j_{1}=0}^{p} h_{j_{3} j_{1}}^{p} \zeta_{j_{1}}^{\left(i_{1}\right)} \zeta_{j_{3}}^{\left(i_{3}\right)}, \quad h_{j_{3} j_{1}}^{p}=\int_{t}^{T} \phi_{j_{1}}\left(s_{3}\right) \int_{s_{3}}^{T} \phi_{j_{3}}(s) F^{p}\left(s_{3}, s\right) d s d s_{3} .
$$

Similar to the above arguing, we obtain that $\Delta_{8}^{\left(i_{1} i_{3}\right)}=0$ with probability 1 . Here we employ the function $K_{p}\left(s, s_{3}\right)=\mathbf{1}_{\left\{s_{3}<s\right\}} F^{p}\left(s_{3}, s\right)$ and the relation

$$
h_{j_{3} j_{1}}^{p}=\int_{[t, T]^{2}} K_{p}\left(s, s_{3}\right) \phi_{j_{1}}\left(s_{3}\right) \phi_{j_{3}}(s) d s d s_{3}, \quad i_{1} \neq i_{3}, \quad i_{1} \neq 0, \quad i_{3} \neq 0 .
$$

In the case $i_{1}=i_{3} \neq 0$, for $h_{j_{1} j_{1}}^{p}$ and $h_{j_{3} j_{1}}^{p}+h_{j_{1} j_{3}}^{p}$, we employ the right hand sides of the formulae (86) and (87), respectively, in which we replace $j_{1}$ by $j_{4}$ and $j_{2}$ by $j_{3}$, respectively.

Let us show that

$$
\lim _{p \rightarrow \infty} \sum_{j_{3}=0}^{p} c_{j_{3} j_{3}}^{p}=0
$$

We have:

$$
c_{j_{3} j_{3}}^{p}=f_{j_{3} j_{3}}^{p}+d_{j_{3} j_{3}}^{p}-g_{j_{3} j_{3}}^{p} .
$$

Similar to the second identity in 83 we obtain

$$
\lim _{p \rightarrow \infty} \sum_{j_{3}=0}^{p} d_{j_{3} j_{3}}^{p}=0
$$

It follows from $(89)$ that

$$
0 \leqslant \lim _{p \rightarrow \infty} \sum_{j_{3}=0}^{p} g_{j_{3} j_{3}}^{p} \leqslant \lim _{p \rightarrow \infty} \frac{(T-t)^{2}}{8(2 p+3)(2 p+1)}=0
$$


that is, identity (97) holds. This completes the proof of identities (74) for the case of Legendre polynomials.

We consider a trigonometric case. According to 63,

$$
a_{j_{4} j_{3}}^{p}=\frac{1}{2} \int_{t}^{T} \phi_{j_{3}}\left(s_{1}\right) \sum_{j_{1}=p+1}^{\infty}\left(\int_{t}^{s_{1}} \phi_{j_{1}}\left(s_{2}\right) d s_{2}\right)^{2} \int_{s_{1}}^{T} \phi_{j_{4}}(s) d s d s_{1} .
$$

Moreover,

$$
\begin{aligned}
& \left|\int_{t}^{s_{1}} \phi_{j}\left(s_{2}\right) d s_{2}\right| \leqslant \frac{K_{0}}{j}, \quad j \neq 0, \quad \int_{s_{1}}^{T} \phi_{0}(s) d s=\frac{T-s_{1}}{\sqrt{T-t}}, \\
& \left|a_{j_{4} j_{3}}^{p}\right| \leqslant \frac{K_{1}}{j_{4}} \sum_{j_{1}=p+1}^{\infty} \frac{1}{j_{1}^{2}} \leqslant \frac{K_{1}}{p j_{4}}\left(j_{4} \neq 0\right), \quad\left|a_{0, j_{3}}^{p}\right| \leqslant \frac{K_{1}}{p} .
\end{aligned}
$$

By $75-77$ and 99 we obtain that $\Delta_{1}^{\left(i_{3} i_{4}\right)}=0$ with probability 1 . In the same way, $\Delta_{2}^{\left(i_{2} i_{4}\right)}=0, \Delta_{4}^{\left(i_{1} i_{3}\right)}=0, \Delta_{6}^{\left(i_{1} i_{3}\right)}=0$ with probability 1 and

$$
\lim _{p \rightarrow \infty} \sum_{j_{3}=0}^{p} a_{j_{3} j_{3}}^{p}=0, \quad \lim _{p \rightarrow \infty} \sum_{j_{3}=0}^{p} b_{j_{3} j_{3}}^{p}=0, \quad \lim _{p \rightarrow \infty} \sum_{j_{3}=0}^{p} f_{j_{3} j_{3}}^{p}=0 .
$$

We consider $\Delta_{3}^{\left(i_{2} i_{4}\right)}$. In this case, as $i_{2}=i_{4} \neq 0$, we employ $84-87$ to get:

$$
\int_{t}^{T} \phi_{j_{4}}(s) \int_{s}^{T} \phi_{j_{1}}\left(s_{2}\right) d s_{2} d s=\frac{\sqrt{2} \sqrt{T-t}}{2 \pi j_{1}} \int_{t}^{T}\left\{\begin{array}{c}
\phi_{j_{4}}(s)\left(1-\cos \frac{2 \pi j_{1}(s-t)}{T-t}\right) d s, \\
\phi_{j_{4}}(s)\left(-\sin \frac{2 \pi j_{1}(s-t)}{T-t}\right) d s,
\end{array}\right.
$$

where $j_{1} \geqslant p+1, j_{4}=0,1, \ldots, p$. By the orthonormality of the trigonometric functions we obtain:

$$
\int_{t}^{T} \phi_{j_{4}}(s) \int_{s}^{T} \phi_{j_{1}}\left(s_{2}\right) d s_{2} d s=\frac{\sqrt{2}(T-t)}{2 \pi j_{1}}\left\{\begin{array}{cc}
1 \text { or } 0 & \text { as } j_{4}=0 \\
0 & \text { otherwise, }
\end{array} \quad j_{1} \geqslant p+1 .\right.
$$

It follows from $(100)$ and $(85)-(87)$ that

$$
\begin{aligned}
& g_{j_{4} j_{2}}^{p}+g_{j_{2} j_{4}}^{p}=\sum_{j_{1}=p+1}^{\infty} \frac{(T-t)^{2}}{2 \pi^{2} j_{1}^{2}}\left\{\begin{array}{cc}
1 \text { or } 0 & \text { as } j_{2}=j_{4}=0 \\
0 & \text { otherwise }
\end{array} \leqslant \frac{K_{1}}{p},\right. \\
& g_{j_{4} j_{4}}^{p}=\sum_{j_{1}=p+1}^{\infty} \frac{(T-t)^{2}}{4 \pi^{2} j_{1}^{2}}\left\{\begin{array}{cc}
1 \text { or } 0 & \text { as } j_{4}=0 \\
0 & \text { otherwise }
\end{array} \frac{K_{1}}{p} .\right.
\end{aligned}
$$

By 101, 102 and 75 we obtain $\Delta_{7}^{\left(i_{2} i_{4}\right)}=0$ and $\Delta_{3}^{\left(i_{2} i_{4}\right)}=0$ with probability 1 as $i_{2}=$ $i_{4} \neq 0$. Similar to the polynomial case, $\Delta_{7}^{\left(i_{2} i_{4}\right)}=0$ and $\Delta_{3}^{\left(i_{2} i_{4}\right)}=0$ with probability 1 as $i_{2} \neq i_{4}$, $i_{2} \neq 0, i_{4} \neq 0$. The same arguing shows that $\Delta_{5}^{\left(i_{1} i_{3}\right)}=0$ with probability 1 .

Taking into considerations $(98)$ and the relations

$$
\lim _{p \rightarrow \infty} \sum_{j_{3}=0}^{p} f_{j_{3} j_{3}}^{p}=\lim _{p \rightarrow \infty} \sum_{j_{3}=0}^{p} d_{j_{3} j_{3}}^{p}=0
$$

implied by the estimates

$$
\left|f_{j j}^{p}\right|+\left|d_{j j}^{p}\right| \leqslant \frac{K_{1}}{p j}, \quad\left|f_{00}^{p}\right|+\left|d_{00}^{p}\right| \leqslant \frac{K_{1}}{p},
$$


we obtain

$$
\lim _{p \rightarrow \infty} \sum_{j_{3}=0}^{p} c_{j_{3} j_{3}}^{p}=-\lim _{p \rightarrow \infty} \sum_{j_{3}=0}^{p} g_{j_{3} j_{3}}^{p}, \quad 0 \leqslant \lim _{p \rightarrow \infty} \sum_{j_{3}=0}^{p} g_{j_{3} j_{3}}^{p} \leqslant \lim _{p \rightarrow \infty} \frac{K_{1}}{p}=0 .
$$

Thus, we arrive to 97 in the trigonometric case. This completes the proof of relations 74 in the trigonometric case and the proof of Theorem 4.

\section{Conclusion}

The results obtained in Theorems 2-4 can be applied to the realization of strong [2, 4] numerical methods of convergence order 1.0 (Milstein method [3]), of convergence orders 1.5 and 2.0 for Itô SDE of form (1) (case of a multi-dimensional Wiener process and a function $B(\mathbf{x}, t)$ depending not only on $t$, but also on $\mathbf{x})$ based on Taylor-Stratonovich expansions [2], [6], 8]. It should be noted that the set of Stratonovich ISIs of multiplicities 1-4 of form (3) employied in constructing of the mentioned numerical methods is universal for both explicit one-step numerical methods and for implicit multi-step and finite-difference (of Runge-Kutta type) modifications.

\section{BIBLIOGRAPHY}

1. I.I. Gikhman, A.V. Skorokhod. The theory of stochastic processes // Kiev, Naukova Dumka (1982). [Classics in Mathematics. Springer, Berlin (2004).]

2. P.E. Kloeden, E. Platen. Numerical solution of stochastic differential equations. Springer-Verlag, Berlin (1992).

3. G.N. Milstein. Numerical integration of stochastic differential equations // Ural Univ. Publ., Sverdlovsk (1988). [Mathematics and its Applications. 313. Kluwer Academic Publ., Dordrecht (1994).]

4. G.N. Milstein, M.V. Tretyakov. Stochastic numerics for mathematical physics Springer-Verlag, Berlin (2004).

5. E. Platen, W. Wagner. On a Taylor formula for a class of Itô processes // Probab. Math. Statist. 3:1, 37-51 (1982).

6. P.E. Kloeden, E. Platen The Stratonovich and Itô-Taylor expansions // Math. Nachr. 151:1, 33-50 (1991).

7. O.Yu. Kulchitski, D.F. Kuznetsov. The unified Taylor-Itô expansion // Zap. Nauch. Seminar. POMI. 244, 168-204 (1997). [J. Math. Sci. 99:2, 1130-1140 (2000).]

8. D.F. Kuznetsov. New representations of the Taylor-Stratonovich expansion // Zap. Nauch. Seminar. POMI. 278, 141-158 (2001). [J. Math. Sci. 118:6, 5586-5596 (2003).]

9. D.F. Kuznetsov. Numerical integration of stochastic differential equations. 2. Polytech. Univ. Publ., St.-Petersburg (2006). (in Russian).

10. P.E. Kloeden, E. Platen, I.W. Wright. The approximation of multiple stochastic integrals // Stoch. Anal. Appl. 10:4, 431-441 (1992).

11. D.F. Kuznetsov. New representations of explicit one-step numerical methods for jump-diffusion stochastic differential equations // Zhurn. Vychisl. Matem. Matem. Fiz. 41:6, 922-937 (2001). [Comp. Math. Math. Phys. 41:6, 874-888 (2001).]

12. E.W. Hobson. The theory of spherical and ellipsoidal harmonics. Cambridge Univ. Press, Cambridge (1931).

13. V.A. Il'in, E.G. Poznyak. Foundations of mathematical analysis. II. Nauka, Moscow (1973). (in Russian).

14. P.K. Suetin. Classical orthogonal polynomials. Fizmatlit, Moscow (2005). (in Russian).

Dmitriy Feliksovich Kuznetsov,

Peter the Great St.Petersburg Polytechnic University,

Polytechnicheskaya str. 29,

195251, Saint-Petersburg, Russia

E-mail: sde_kuznetsov@inbox.ru 